\title{
Strong vertices of doubly heavy spin-3/2-spin-1/2 baryons with light mesons in light-cone QCD sum rules
}

\author{
T. M. Aliev®* \\ Physics Department, Middle East Technical University, Ankara 06800, Turkey \\ K. Şimşek $\oplus^{\dagger}$ \\ Department of Physics \& Astronomy, Northwestern University, Evanston, Illinois 60208, USA
}

(Received 4 February 2021; accepted 8 March 2021; published 30 March 2021)

\begin{abstract}
In this paper, we analyze the vertices of doubly heavy spin-3/2-spin-1/2 baryons with light mesons within the method of light-cone QCD sum rules. These vertices are parametrized in terms of one (three) coupling constant(s) for the pseudoscalar (vector) mesons. The said coupling constants are calculated for all possible transitions. The results presented here can serve as useful information in experimental as well as theoretical studies of the properties of doubly heavy baryons.
\end{abstract}

DOI: 10.1103/PhysRevD.103.054044

\section{INTRODUCTION}

The quark model predicts the existence of many doubly heavy baryons. $\Xi_{c c}^{+}$was discovered in experiments in the decay modes $\Lambda_{c}^{+} K^{-} \pi^{+}$and $p D^{+} K^{-}$with the mass $m_{\Xi_{c c}^{+}}=3518.7 \pm 17 \mathrm{MeV}$ by the SELEX Collaboration at Fermilab $[1,2]$ and $\Xi_{c c}^{++}$was observed by the LHCb Collaboration [3,4] in the $\Lambda_{c}^{+} K^{-} \pi^{+} \pi^{+}$mass spectrum and confirmed via measuring $\Xi_{c c}^{++} \rightarrow \Xi_{c}^{+} \pi^{+}$with the mass $m_{\Xi_{c c}^{++}}=3621.24 \pm 0.65 \pm 0.31 \mathrm{MeV}$.

The main effort of experimentalists is focused on the discovery of other members of doubly heavy baryons predicted by the quark model. From the theoretical point of view, these baryons provide an excellent laboratory to study their electromagnetic, weak, and strong decays for a better understanding their quark structure, check the predictions of heavy-quark symmetry, as well as gain information about perturbative and nonperturbative aspects of QCD.

The strong coupling constants of doubly heavy baryons with light mesons are the main ingredients of their decays. The formation of hadrons takes place at a low-energy domain which belongs to the nonperturbative region of QCD. Thus, for the determination of the strong coupling constants of doubly heavy baryons with light mesons, we

\footnotetext{
taliev@metu.edu.tr

†ksimsek@u.northwestern.edu
}

Published by the American Physical Society under the terms of the Creative Commons Attribution 4.0 International license. Further distribution of this work must maintain attribution to the author(s) and the published article's title, journal citation, and DOI. Funded by SCOAP . need some nonperturbative method. At the hadronic scale, one should refer to nonperturbative methods in QCD as the strong coupling constant is large and hence perturbative theory becomes invalid. The method of the QCD sum rules [5] has proved to be one of the most powerful among all other nonperturbative methods in studying the properties of hadrons. The most advanced version of the method appears to be the light-cone formalism. The light-cone sum rules (LCSR) was introduced in [6-8] and then rapidly developed by applying it to the wide spectrum of problems of hadron physics (see, for example, the reviews [9-11] and references therein). Here, we would like to mention that the strong coupling constants for $D^{*} D \pi$ and $B^{*} B \pi$ within the LCSR were first estimated in [12].

In the LCSR, the operator product expansion (OPE) is performed over a twist near the light cone, $x^{2} \sim 0$. In this case, there appear matrix elements of nonlocal operators between a one-particle baryon state and the vacuum. These matrix elements are parametrized in terms of distribution amplitudes (DAs).

Properties of doubly heavy baryons have been studied within the frameworks of lattice QCD [13], quark spin symmetry [14,15], and QCD sum rules [16-20]. The strong coupling constants of doubly heavy spin- $1 / 2$ baryons are examined in [20-23]. The strong coupling constants of light vector mesons with doubly heavy spin-3/2 baryons are estimated in [24]. We would like to emphasize the work [12] where the $D^{*} D \pi$ and $B^{*} B \pi$ strong coupling constants are calculated within the LCSR formalism, which is related to the present work.

This paper is organized as follows. In Sec. II, we derive the LCSR for the coupling constants of the light mesons with doubly heavy baryons in the spin-3/2-to-spin- $1 / 2$ transitions. In Sec. III, the numerical analysis of the 
obtained sum rules is performed. Section IV contains our conclusion.

\section{LIGHT-CONE SUM RULES FOR THE SPIN-3/2-SPIN-1/2 DOUBLY HEAVY BARYONS WITH LIGHT MESONS}

To determine the coupling constants of the pseudoscalar and vector mesons with the spin-3/2-to-spin-1/2 doubly heavy baryons within the LCSR, we consider the following correlation function:

$$
\Pi_{\mu}=i \int d^{4} x e^{i p x}\left\langle\mathcal{M}(q)\left|T\left\{\eta_{\mu}(x) \bar{\eta}(0)\right\}\right| 0\right\rangle
$$

where $\mathcal{M}(q)$ is a light meson with 4-momentum $q$, and $\eta_{\mu}$ and $\eta$ denote the interpolating current of the corresponding spin- $1 / 2$ and spin-3/2 doubly heavy baryons, respectively. The most general form of the interpolating current for spin$1 / 2$ doubly heavy baryons can be written as

$\eta_{\mu}=N \epsilon^{a b c}\left[\left(q^{a \mathrm{~T}} B Q^{b}\right) Q^{\prime c}+\left(q^{a \mathrm{~T}} B Q^{\prime b}\right) Q^{c}+\left(Q^{a \mathrm{~T}} B Q^{\prime b}\right) q^{c}\right]$

$\eta^{(S)}=\frac{1}{\sqrt{2}} \epsilon^{a b c} \sum_{i=1}^{2}\left[\left(Q^{a \mathrm{~T}} A_{1}^{i} q^{b}\right) A_{2}^{i} Q^{\prime c}+\left(Q \leftrightarrow Q^{\prime}\right)\right]$

$$
\begin{aligned}
\eta^{(A)}= & \frac{1}{\sqrt{6}} \epsilon^{a b c} \sum_{i=1}^{2}\left[2\left(Q^{a} A_{1}^{i} Q^{\prime b}\right) A_{2}^{i} q^{c}+\left(Q^{a \mathrm{~T}} A_{1}^{i} q^{b}\right) A_{2}^{i} Q^{\prime c}\right. \\
& \left.-\left(Q^{\prime a \mathrm{~T}} A_{1}^{i} q^{b}\right) A_{2}^{i} Q^{c}\right]
\end{aligned}
$$

where $N=\sqrt{1 / 3}(\sqrt{2 / 3})$ for identical (different) quarks and

$$
A_{1}^{1}=C, A_{2}^{1}=\gamma_{5}, A_{1}^{2}=C \gamma_{5}, A_{2}^{2}=\beta I, B=C \gamma_{\mu} .
$$

Here, $\mathrm{T}$ is the transpose, $C$ is the charge conjugation operator, and $\beta$ is an arbitrary parameter, for which $\beta=-1$ corresponds to the Ioffe current. The superscripts $S$ and $A$ in Eqs. (3) and (4) indicate symmetric and antisymmetric currents with respect to the interchange of heavy quarks.

In order to obtain the LCSR for appropriate quantities, the correlation function is calculated in two different regions: First, in terms of hadrons and second, in terms of quark-gluon degrees of freedom in the deep Euclidean region by using the OPE. Then, by using the dispersion relation, these two representations are matched and as a result, the desired sum rules are obtained.

The hadronic representation of the correlation function can be obtained by inserting the complete set of baryon states carrying the same quantum numbers as the interpolating current. Isolating the ground-state contributions, we obtain
$\Pi_{\mu}=\frac{\left\langle 0\left|\eta_{\mu}\right| B^{*}\left(p_{2}\right)\right\rangle\left\langle\mathcal{M}(q) B^{*}\left(p_{2}\right) \mid B\left(p_{1}\right)\right\rangle\left\langle B\left(p_{1}\right)|\bar{\eta}(0)| 0\right\rangle}{\left(p_{2}^{2}-m_{2}^{2}\right)\left(p_{1}^{2}-m_{1}^{2}\right)}$

$+\cdots$

where $B^{*}\left(p_{2}\right)$ and $B\left(p_{1}\right)$ denote the spin-3/2 and spin- $1 / 2$ doubly heavy baryons and $m_{2}$ and $m_{1}$ their mass, respectively. In Eq. (6), $\cdots$ describes the contribution of higher states and the continuum.

For the calculation of the phenomenological side of the correlation function, the matrix elements $\left\langle 0\left|\eta_{\mu}\right| B^{*}\left(p_{2}\right)\right\rangle$, $\left\langle\mathcal{M}(q) B^{*}\left(p_{2}\right) \mid B\left(p_{1}\right)\right\rangle$, and $\left\langle B\left(p_{1}\right)|\eta| 0\right\rangle$ are needed. These matrix elements are determined in the following way:

$$
\begin{aligned}
\left\langle 0|\eta| B\left(p_{1}\right)\right\rangle & =\lambda_{1} u\left(p_{1}\right) \\
\left\langle 0\left|\eta_{\mu}\right| B^{*}\left(p_{2}\right)\right\rangle & =\lambda_{2} u_{\mu}\left(p_{2}\right) \\
\left\langle\mathcal{P}(q) B^{*}\left(p_{2}\right) \mid B\left(p_{1}\right)\right\rangle & =g \bar{u}_{\alpha}\left(p_{2}\right) u\left(p_{1}\right) q^{\alpha} .
\end{aligned}
$$

The matrix element $\left\langle V(q) B^{*}\left(p_{2}\right) \mid B\left(p_{1}\right)\right\rangle$ is parametrized in terms of three couplings as follows [25]:

$$
\begin{aligned}
\left\langle V(q) B^{*}\left(p_{2}\right) \mid B\left(p_{1}\right)\right\rangle= & \bar{u}_{\alpha}\left(p_{2}\right)\left[g_{1}\left(\varepsilon_{\alpha} \not 1-q_{\alpha} \phi\right) \gamma_{5}\right. \\
& +g_{2}\left(P \cdot q \varepsilon_{\alpha}-P \cdot \varepsilon q_{\alpha}\right) \gamma_{5} \\
& \left.+g_{3}\left(q \cdot \varepsilon q_{\alpha}-q^{2} \varepsilon_{\alpha}\right) \gamma_{5}\right] u\left(p_{1}\right)
\end{aligned}
$$

where $u\left(p_{1}\right)$ is the Dirac bispinor for spin- $1 / 2$ baryons while $u_{\alpha}\left(p_{2}\right)$ is the Rarita-Schwinger spinor for spin-3/2 baryons, $\varepsilon_{\mu}$ is the polarization four-vector of the light vector meson, $P=\left(p_{1}+p_{2}\right) / 2$, and $q=p_{1}-p_{2}$. In the following discussions, we denote $p_{2}=p$ and $p_{1}=p+q$ as well as impose the on-shell condition for the vector meson, namely, $q^{2}=m_{V}^{2}$, and the gauge condition $q \cdot \varepsilon=0$.

Taking into account Eqs. (7)-(10) in Eq. (6) and using the completeness conditions for Dirac and RaritaSchwinger spinors, which read

$$
\begin{gathered}
\sum_{s} u(p) \bar{u}(p)=\not p+m \\
\sum_{s} u_{\alpha}(p) \bar{u}_{\beta}(p)=-(\not p+m)\left(g_{\alpha \beta}-\frac{1}{3} \gamma_{\alpha} \gamma_{\beta}+\frac{2}{3} \frac{p_{\alpha} p_{\beta}}{m^{2}}\right. \\
\left.+\frac{p_{\alpha} \gamma_{\beta}-p_{\beta} \gamma_{\alpha}}{3 m}\right)
\end{gathered}
$$

one can easily get the expressions for the strong coupling constants of the light mesons with the doubly heavy baryons. Before moving to the next calculations, here we would like to bring the attention of the reader to the existence of two problems: 
(i) The negative-parity spin- $1 / 2$ baryon $B^{-}(p)$ contributes to the matrix element $\left\langle 0\left|\eta_{\mu}\right| B^{-}(p)\right\rangle$. From general considerations, this matrix element can be parametrized as

$$
\left\langle 0\left|\eta_{\mu}\right| B^{-}(p)\right\rangle=A\left(\gamma_{\mu}-\frac{4}{m} p_{\mu}\right) u(p, s) .
$$

From this equation, it follows that the structures proportional to $\gamma_{\mu}$ and $p_{\mu}$ contain the contributions coming not only from spin-3/2 baryons, but also from spin- $1 / 2$ baryons, which should be eliminated. Therefore, we will discard these structures in the next discussions. From Eq. (12), it follows that only the structures proportional to $g_{\alpha \beta}$ contain solely the contribution of the spin-3/2 state.

(ii) Not all Lorentz structures are independent. This problem can be overcome by using a specific order of Dirac matrices. For the calculations of the strong coupling constants of pseudoscalar (vector) mesons with the spin- $3 / 2$ and spin- $1 / 2$ baryons, the ordered Dirac matrices are chosen in the form $\not \not p \not \gamma_{\mu}\left(\gamma_{\mu} \phi \phi \not p \gamma_{5}\right)$.

Taking into account these facts and using Eqs. (7)-(10) in the phenomenological parts of the correlation functions, we finally get

$\Pi_{\mu}^{(\mathcal{P})}=\frac{g \lambda_{1} \lambda_{2} \not p \not p q_{\mu}}{\left(m_{2}^{2}-p^{2}\right)\left[m_{1}^{2}-(p+q)^{2}\right]}+$ other structures

and

$$
\begin{aligned}
\Pi_{\mu}^{(\mathcal{V})}= & \frac{\lambda_{1} \lambda_{2}}{\left[m_{1}^{2}-(p+q)^{2}\right]\left[m_{2}^{2}-p^{2}\right]}\left[-g_{1}\left(m_{1}+m_{2}\right) \notin \not p \gamma_{5} q_{\mu}\right. \\
& +g_{2} \not \not p \gamma_{5} p \cdot \varepsilon q_{\mu}+g_{3} m_{V}^{2} \not \not p \gamma_{5} \varepsilon_{\mu} \\
& + \text { other structures]. }
\end{aligned}
$$

To obtain the LCSR for the aforementioned coupling constants, we need to calculate the correlation function from the QCD side and choose the coefficients of the same structures and then match with the results from the hadronic part. The expression of the correlation function is obtained by using OPE in the deep Euclidean region, $p^{2} \ll 0$ and $(p+q)^{2} \ll 0$. Using the Wick theorem, from Eq. (1) one can get

$$
\begin{aligned}
\Pi_{\mu}^{(S)}= & \sqrt{\frac{1}{3}} \epsilon^{a b c} \epsilon^{a^{\prime} b^{\prime} c^{\prime}} \int d^{4} x e^{i p x} \sum_{i=1}^{2} B_{\alpha \beta}\left(\tilde{A}_{2}^{i}\right)_{\gamma^{\prime} \rho^{\prime}}\left(\tilde{A}_{1}^{i}\right)_{\alpha^{\prime} \beta^{\prime}} \\
& \times\langle\mathcal{M}(q)|\left(-S_{Q^{\prime} \gamma \gamma^{\prime}}^{c c^{\prime}} S_{Q \beta \beta^{\prime}}^{b a^{\prime}} q_{\alpha}^{a} \bar{q}_{\alpha^{\prime}}^{b^{\prime}}-S_{Q \gamma^{\prime}}^{c c^{\prime}} S_{Q^{\prime} \beta \beta^{\prime}}^{b a^{\prime}} q_{\alpha}^{a} \bar{q}_{\alpha^{\prime}}^{b^{\prime}}\right. \\
& +S_{Q \beta \gamma^{\prime}}^{b c^{\prime}} S_{Q^{\prime} \gamma \beta^{\prime}}^{c a^{\prime}} q_{\alpha}^{a} \bar{q}_{\alpha^{\prime}}^{b^{\prime}}+S_{Q^{\prime} \beta \gamma^{\prime}}^{b c^{\prime}} S_{Q \gamma \gamma^{\prime}}^{c a^{\prime}} q_{\alpha}^{a} \bar{q}_{\alpha^{\prime}}^{b^{\prime}} \\
& \left.-S_{Q^{\prime} \beta \gamma^{\prime}}^{b c^{\prime}} S_{Q \alpha \beta^{\prime}}^{a a^{\prime}} q_{\gamma}^{c} \bar{q}_{\alpha^{\prime}}^{b^{\prime}}+S_{Q \alpha \gamma^{\prime}}^{a c^{\prime}} S_{Q^{\prime} \beta \beta^{\prime}}^{b a^{\prime}} q_{\gamma}^{c} \bar{q}_{\alpha^{\prime}}^{b^{\prime}}\right)|0\rangle
\end{aligned}
$$

and

$$
\begin{aligned}
\Pi_{\mu}^{(A)}= & \sqrt{\frac{1}{3}} \epsilon^{a b c} \epsilon^{a^{\prime} b^{\prime} c^{\prime}} \int d^{4} x e^{i p x} \sum_{i=1}^{2} B_{\alpha \beta}\left(\tilde{A}_{2}^{i}\right)_{\gamma^{\prime} \rho^{\prime}}\left(\tilde{A}_{1}^{i}\right)_{\alpha^{\prime} \beta^{\prime}} \\
& \times\langle\mathcal{M}(q)|\left(-S_{Q^{\prime} \gamma \gamma^{\prime}}^{c c^{\prime}} S_{Q \beta \beta^{\prime}}^{b a^{\prime}} q_{\alpha}^{a} \bar{q}_{\alpha^{\prime}}^{b^{\prime}}+S_{Q^{\prime} \beta \beta^{\prime}}^{b a^{\prime}} S_{Q \gamma \gamma^{\prime}}^{c c^{\prime}} q_{\alpha}^{a} \bar{q}_{\alpha^{\prime}}^{b^{\prime}}\right. \\
& +2 S_{Q^{\prime} \beta \alpha^{\prime}}^{b b^{\prime}} S_{Q \alpha \beta^{\prime}}^{a a^{\prime}} q_{\gamma}^{c} \bar{q}_{\gamma^{\prime}}^{c^{\prime}}+2 S_{Q^{\prime} \gamma \alpha^{\prime}}^{c b^{\prime}} S_{Q \beta \beta^{\prime}}^{b a^{\prime}} q_{\alpha}^{a} \bar{q}_{\gamma^{\prime}}^{c^{\prime}} \\
& -2 S_{Q^{\prime} \beta \alpha^{\prime}}^{b b^{\prime}} S_{Q \gamma \beta^{\prime}}^{c a^{\prime}} q_{\alpha}^{a} \bar{q}_{\gamma^{\prime}}^{c^{\prime}}-S_{Q \beta \gamma^{\prime}}^{b c^{\prime}} S_{Q^{\prime} \gamma \beta^{\prime}}^{c a^{\prime}} q_{\alpha}^{a} \bar{q}_{\alpha^{\prime}}^{b^{\prime}} \\
& +S_{Q^{\prime} \beta \gamma^{\prime}}^{b c^{\prime}} S_{Q \gamma \beta^{\prime}}^{c a^{\prime}} q_{\alpha}^{a} \bar{q}_{\alpha^{\prime}}^{b^{\prime}}-S_{Q^{\prime} \beta \gamma^{\prime}}^{b c^{\prime}} S_{Q \alpha \beta^{\prime}}^{a a^{\prime}} q_{\gamma}^{c} \bar{q}_{\alpha^{\prime}}^{b^{\prime}} \\
& \left.-S_{Q \alpha \gamma^{\prime}}^{a c^{\prime}} S_{Q^{\prime} \beta \beta^{\prime}}^{b a^{\prime}} q_{\gamma}^{c} \bar{q}_{\alpha^{\prime}}^{b^{\prime}}\right)|0\rangle .
\end{aligned}
$$

Here, $S_{Q}$ is the heavy-quark propagator and $\tilde{A}_{k}^{i}=\gamma^{0} A_{k}^{i \dagger} \gamma^{0}$. The heavy-quark propagator in the presence of an external background field is given in the coordinate representation by

$$
\begin{aligned}
S_{Q \alpha \beta}^{a a^{\prime}}(x)= & \frac{m_{Q}^{2}}{4 \pi}\left[\frac{i \chi K_{2}\left(m_{Q} \sqrt{-x^{2}}\right)}{\left(\sqrt{-x^{2}}\right)^{2}}+\frac{K_{1}\left(m_{Q} \sqrt{-x^{2}}\right)}{\sqrt{-x^{2}}}\right]_{\alpha \beta} \\
& \times \delta^{a a^{\prime}}-\frac{g_{s}}{16 \pi^{2}} m_{Q} \int_{0}^{1} d u \\
& \times\left[\frac{i K_{1}\left(m_{Q} \sqrt{-x^{2}}\right)}{\sqrt{-x^{2}}}\left(u \chi \sigma_{\lambda \tau}+\bar{u} \sigma_{\lambda \tau} \chi x\right)\right. \\
& \left.+K_{0}\left(m_{Q} \sqrt{-x^{2}}\right) \sigma_{\lambda \tau}\right]_{\alpha \beta} G^{(n) \lambda \tau}\left(\frac{\lambda^{n}}{2}\right)^{a a^{\prime}}
\end{aligned}
$$

where $G_{\lambda \tau}^{(n)}$ is the gluon field strength tensor, the $\lambda^{(n)}$ are the Gell-Mann matrices, and the $K_{i}\left(m_{Q} \sqrt{-x^{2}}\right)$ are the modified Bessel functions of the second kind.

Using the Fiertz identities

$$
\begin{gathered}
q_{\alpha}^{b} \bar{q}_{\beta}^{b^{\prime}}=-\frac{1}{12}\left(\Gamma_{i}\right)_{\alpha \beta} \delta^{b b^{\prime}} \bar{q} \Gamma_{i} q \\
q_{\alpha}^{b} \bar{q}_{\beta}^{b^{\prime}} G_{\lambda \tau}^{(n)}=-\frac{1}{16}\left(\frac{\lambda^{(n)}}{2}\right)^{b b^{\prime}}\left(\Gamma_{i}\right)_{\alpha \beta} \bar{q} \Gamma_{i} G_{\lambda \tau}^{(n)} q
\end{gathered}
$$

from Eqs. (16) and (17), it follows that, in the calculation of the correlation functions from the QCD side, the matrix elements $\left\langle\mathcal{M}(q)\left|\bar{q} \Gamma_{i} q\right| 0\right\rangle$ and $\left\langle\mathcal{M}(q)\left|\bar{q} \Gamma_{i} G_{\lambda \tau}^{(n)} q\right| 0\right\rangle$ appear, where $\left\{\Gamma_{i}\right\}$ is the full set of Dirac matrices,

$$
\Gamma_{i}=\left\{I, \gamma_{5}, \gamma_{\mu}, i \gamma_{\mu} \gamma_{5}, \frac{1}{\sqrt{2}} \sigma_{\mu \nu}\right\}_{i=1}^{5} .
$$

These matrix elements are the main nonperturbative input parameters of the LCSR and they are expressed in terms of light meson DAs of different twists. The expressions of 
these matrix elements in terms of meson DAs are found in [26-31] and for completeness, we present their expressions in the Appendix A.

Inserting Eqs. (19) and (20) into (16) and (17), performing a double Borel transformation over the variables $-p^{2}$ and $-(p+q)^{2}$ in both representations of the correlation function, and choosing the coefficients of the corresponding structures, we get the following sum rules for the desired coupling constants:

$$
g=\frac{1}{\lambda_{1} \lambda_{2}} e^{m_{1}^{2} / M_{1}^{2}+m_{2}^{2} / M_{2}^{2}} \Pi^{\text {theo }(S, A)}
$$

$$
\begin{gathered}
g_{1}=-\frac{1}{\lambda_{1} \lambda_{2}\left(m_{1}+m_{2}\right)} e^{m_{1}^{2} / M_{1}^{2}+m_{2}^{2} / M_{2}^{2} \Pi_{1}^{\text {theo }(S, A)}} \\
g_{2}=\frac{1}{\lambda_{1} \lambda_{2}} e^{m_{1}^{2} / M_{1}^{2}+m_{2}^{2} / M_{2}^{2} \Pi_{2}^{\text {theo }(S, A)}} \\
g_{3}=\frac{1}{\lambda_{1} \lambda_{2} m_{V}^{2}} e^{m_{1}^{2} / M_{1}^{2}+m_{2}^{2} / M_{2}^{2}} \Pi_{3}^{\text {theo }(S, A)}
\end{gathered}
$$

where $\Pi^{\text {theo }(A)}=0=\Pi_{i}^{\text {theo }(A)}$ for all the coupling constants and

$$
\begin{gathered}
\Pi^{\text {theo }(S)}=\frac{i m_{Q}^{2} m_{Q^{\prime}}^{2}}{12 \sqrt{3} \pi^{4} M^{4}}\left\{6(-1+\beta) f_{\mathcal{P}} \mathcal{I}_{22}\left[\phi_{\mathcal{P}}(u)\right]-(1+\beta) \mu_{\mathcal{P}}\left(-1+\tilde{\mu}_{\mathcal{P}}^{2}\right) \mathcal{I}_{12}\left[\phi_{\sigma}(u)\right]\right\} \\
\begin{aligned}
\Pi_{1}^{\text {theo }(S)}=- & \frac{i m_{Q}^{2} m_{Q^{\prime}}^{2}}{32 \sqrt{3} \pi^{4} M^{4}}\left\{16(-1+\beta) f_{V} m_{V} \mathcal{I}_{22}\left[\phi_{3}^{\perp}(u)\right]+f_{V}^{T}\left[16 M^{2}(1+2 \beta) \mathcal{I}_{12}\left[\phi_{2}^{\perp}(u)\right]\right.\right. \\
+ & \left.m_{V}^{2}\left(8 i(1+\beta) \mathcal{I}_{12}\left[\mathcal{H}\left[1, \phi_{2}^{\perp}(u)\right]-\mathcal{H}\left[1, \psi \psi_{4}^{\perp}(u)\right]\right)+M^{2}(1+2 \beta) \mathcal{I}_{12}^{2}\left[\phi_{4}^{\perp}(u)\right]\right]\right\} \\
\Pi_{2}^{\text {theo }(S)}= & \frac{m_{Q}^{2} m_{Q^{\prime}}^{2}}{2 \sqrt{3} \pi^{4} M^{6}}\left\{i(1+\beta) f_{V}^{T} \mathcal{I}_{12}\left[\mathcal{H}\left[1, \phi_{2}^{\perp}(u)\right]-2 \mathcal{H}\left[2, \phi_{3}^{\perp}(u)\right]+\mathcal{H}\left[2, \psi_{4}^{\perp}(u)\right]\right]\right. \\
+ & \left.2(-1+\beta) f_{V} m_{V} \mathcal{I}_{22}\left[\mathcal{H}\left[1, \phi_{2}^{\|}(u)\right]-\mathcal{H}\left[1, \phi_{3}^{\perp}(u)\right]\right]\right\} \\
\Pi_{3}^{\text {theo }(S)}= & -\frac{i m_{Q}^{2} m_{Q^{\prime}}^{2}}{32 \sqrt{3} \pi^{4} M^{4}}\left\{32 i(-1+\beta) f_{V} m_{V} \mathcal{I}_{22}\left[\mathcal{H}\left[1, \phi_{2}^{\|}(u)\right]-\mathcal{H}\left[1, \phi_{3}^{\perp}(u)\right]\right]\right. \\
& +f_{V}^{T}\left(-16(1+\beta) \mathcal{I}_{12}\left[\mathcal{H}\left[2, \phi_{2}^{\perp}(u)\right]-2 \mathcal{H}\left[2, \phi_{3}^{\|}(u)\right]+\mathcal{H}\left[2, \psi_{4}^{\perp}(u)\right]\right]\right. \\
& \left.\left.+16(1+2 \beta) M^{2} \mathcal{I}_{12}\left[\mathcal{H}\left[1, \phi_{2}^{\perp}(u)\right]\right]+(1+2 \beta) m_{V}^{2} M^{2} I_{12}\left[\phi_{4}^{\perp}(u)\right]\right)\right\}
\end{aligned}
\end{gathered}
$$

where we have defined the integrals and operators

$$
\begin{array}{r}
\mathcal{I}_{i j}[f(u)]:=\int d u \int d^{4} x e^{i(p+\bar{u} q) \cdot x} K_{i} K_{j} f(u) \\
\mathcal{I}_{i j}^{2}[f(u)]:=\int d u \int d^{4} x e^{i(p+\bar{u} q) \cdot x} K_{i} K_{j} f(u) x^{2} \\
\mathcal{I}_{i j}^{4}[f(u)]:=\int d u \int d^{4} x e^{i(p+\bar{u} q) \cdot x} K_{i} K_{j} f(u) x^{4} \\
\mathcal{H}[n, f(u)]:=i^{n} \int_{0}^{u} d v_{n} \cdots \int_{0}^{v_{3}} d v_{2} \int_{0}^{v_{2}} d v_{1} f\left(v_{1}\right)
\end{array}
$$

and we have introduced the shorthand notation

$$
K_{i}:=\frac{K_{i}\left(m_{Q} \sqrt{-x^{2}}\right)}{\left(\sqrt{-x^{2}}\right)^{i}}, \quad K_{j}:=\frac{K_{j}\left(m_{Q^{\prime}} \sqrt{-x^{2}}\right)}{\left(\sqrt{-x^{2}}\right)^{j}}
$$

Here, for the sake of simplicity, we omit the contributions coming from the matrix elements of three-particle nonlocal operators between the vacuum and one-particle meson state but we include them in our numerical analysis. The details of the calculations of the correlation function from the QCD side are presented in Appendix B.

Since the masses of the spin-3/2 and spin-1/2 doubly heavy baryons are close to each other, we put $M_{1}^{2}=M_{2}^{2}=$ $2 M^{2}$ here and in all the following discussions.

\section{NUMERICAL ANALYSIS}

In this section, we perform the numerical analysis of the LCSR for the coupling constants $g$ and $g_{i}$ of the light mesons $\pi, K, \rho$, and $K^{*}$ in the transition of the spin-3/2to-spin- $1 / 2$ doubly heavy baryons, namely, $\Xi_{c c}^{*}, \Xi_{b b}^{*}, \Xi_{b c}^{*}$, $\Omega_{c c}^{*}, \Omega_{b b}^{*}, \Omega_{b c}^{*}$ to $\Xi_{c c}, \Xi_{b b}, \Xi_{b c}, \Omega_{c c}, \Omega_{b b}$, and $\Omega_{b c}$ by using Package $X$ [32]. The LCSR for the coupling constants $g, g_{1}, g_{2}$, and $g_{3}$ involve various input 
TABLE I. Some of the values of the input parameters entering the sum rules. All the masses and decay constants are in units of $\mathrm{GeV}$.

\begin{tabular}{lclccc}
\hline \hline Parameter & Value & Parameter & Value & Parameter & Value \\
\hline$m_{s}(1 \mathrm{GeV})$ & 0.137 & $m_{\Xi_{c c}}[16]$ & 3.72 & $\lambda_{\Xi_{c c}}[16]$ & 0.16 \\
$m_{c}$ & 1.4 & $m_{\Xi_{b b}}[16]$ & 9.96 & $\lambda_{\Xi_{b b}}[16]$ & 0.44 \\
$m_{b}$ & 4.7 & $m_{\Xi_{b c}}[16]$ & 6.72 & $\lambda_{\Xi_{b c}}[16]$ & 0.28 \\
$m_{\rho}$ & 0.770 & $m_{\Omega_{c c}}[16]$ & 3.73 & $\lambda_{\Omega_{c c}}[16]$ & 0.18 \\
$f_{\rho}$ & 0.216 & $m_{\Omega_{b b}}[16]$ & 9.97 & $\lambda_{\Omega_{b b}}[16]$ & 0.45 \\
$f_{\rho}^{T}$ & 0.165 & $m_{\Omega_{b c}}[16]$ & 6.75 & $\lambda_{\Omega_{b c}}[16]$ & 0.29 \\
$m_{K^{*}}$ & 0.892 & $m_{\Xi_{c c}^{*}}[17]$ & 3.69 & $\lambda_{\Xi_{c c}^{*}}[17]$ & 0.12 \\
$f_{K^{*}}$ & 0.220 & $m_{\Xi_{b b}^{*}}[17]$ & 10.4 & $\lambda_{\Xi_{b b}^{*}}[17]$ & 0.22 \\
$f_{K^{*}}^{T}$ & 0.185 & $m_{\Xi_{b c}^{*}}[17]$ & 7.25 & $\lambda_{\Xi_{b c}^{*}}[17]$ & 0.15 \\
$m_{\pi}$ & 0.135 & $m_{\Omega_{c c}^{*}}[17]$ & 3.78 & $\lambda_{\Omega_{c c}^{*}}[17]$ & 0.14 \\
$f_{\pi}$ & 0.131 & $m_{\Omega_{b b}^{*}}[17]$ & 10.5 & $\lambda_{\Omega_{b b}^{*}}[17]$ & 0.25 \\
$m_{K}$ & 0.495 & $m_{\Omega_{b c}^{*}}[17]$ & 7.3 & $\lambda_{\Omega_{b c}^{*}}[17]$ & 0.17 \\
$f_{K}$ & 0.160 & & & & \\
\hline \hline
\end{tabular}

TABLE II. The working region of the parameters $M^{2}$ and $s_{0}$ for the transitions considered in our work. Here, $\mathcal{M}_{1}=\pi^{ \pm}$or $\rho^{ \pm}$and $\mathcal{M}_{2}=K$ or $K^{*}$.

\begin{tabular}{lcc}
\hline \hline Channel & $M^{2}\left(\mathrm{GeV}^{2}\right)$ & $s_{0}\left(\mathrm{GeV}^{2}\right)$ \\
\hline$\Xi_{c c}^{*} \rightarrow \Xi_{c c} \mathcal{M}_{1}$ & $3.0 \leq M^{2} \leq 4.5$ & $18 \pm 4$ \\
$\Xi_{b b}^{*} \rightarrow \Xi_{b b} \mathcal{M}_{1}$ & $8 \leq M^{2} \leq 12$ & $110 \pm 10$ \\
$\Xi_{b c}^{*} \rightarrow \Xi_{b c} \mathcal{M}_{1}$ & $6 \leq M^{2} \leq 8$ & $60 \pm 5$ \\
$\Omega_{c c}^{*} \rightarrow \Xi_{c c} \mathcal{M}_{2}$ & $3.0 \leq M^{2} \leq 4.5$ & $18 \pm 4$ \\
$\Omega_{b b}^{*} \rightarrow \Xi_{b b} \mathcal{M}_{2}$ & $8 \leq M^{2} \leq 12$ & $110 \pm 10$ \\
$\Omega_{b c}^{*} \rightarrow \Xi_{b c} \mathcal{M}_{2}$ & $6 \leq M^{2} \leq 8$ & $60 \pm 5$ \\
\hline \hline
\end{tabular}

parameters such as quark masses, the masses and decay constants of the light mesons, and the masses and residues of the said doubly heavy baryons. Some of these parameters are presented in Table I. Another set of essential input parameters is meson DAs of different twists, which are given in Appendix A.

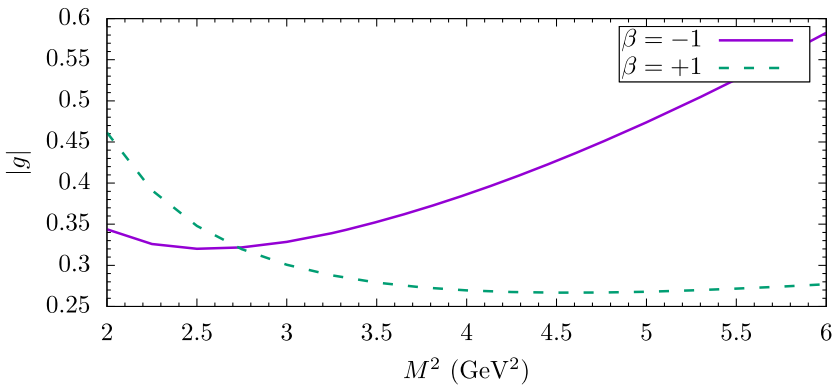

FIG. 1. The dependence of the coupling constant $|g|$ for the transition $\Xi_{c c}^{*++} \rightarrow \Xi_{c c}^{+} \pi^{+}$on $M^{2}$ at shown values of $\beta$ and the fixed value of $s_{0}=18 \mathrm{GeV}^{2}$.

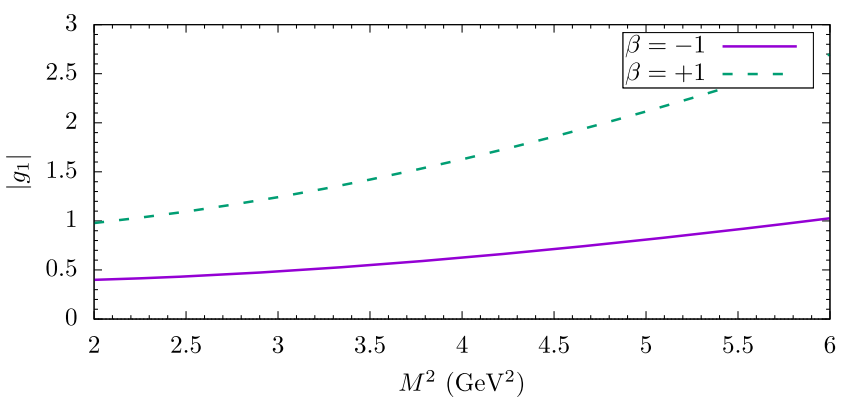

FIG. 2. The dependence of the coupling constant $\left|g_{1}\right|$ for the transition $\Xi_{c c}^{*++} \rightarrow \Xi_{c c}^{+} \rho^{+}$on $M^{2}$ at shown values of $\beta$ and the fixed value of $s_{0}=18 \mathrm{GeV}^{2}$.

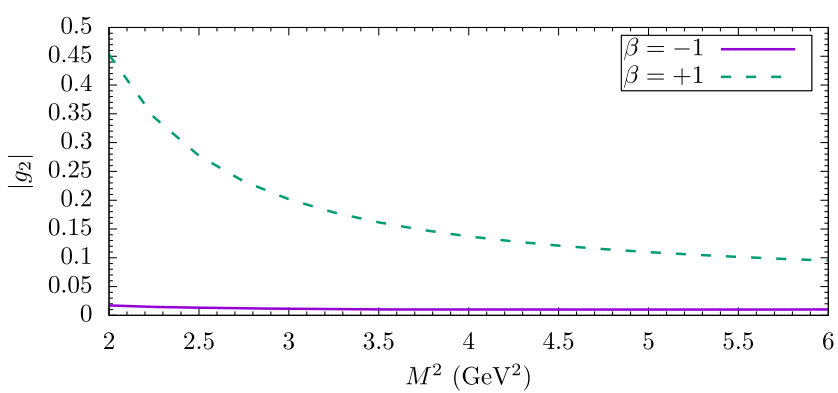

FIG. 3. The same as Fig. 2 but for $\left|g_{2}\right|$.

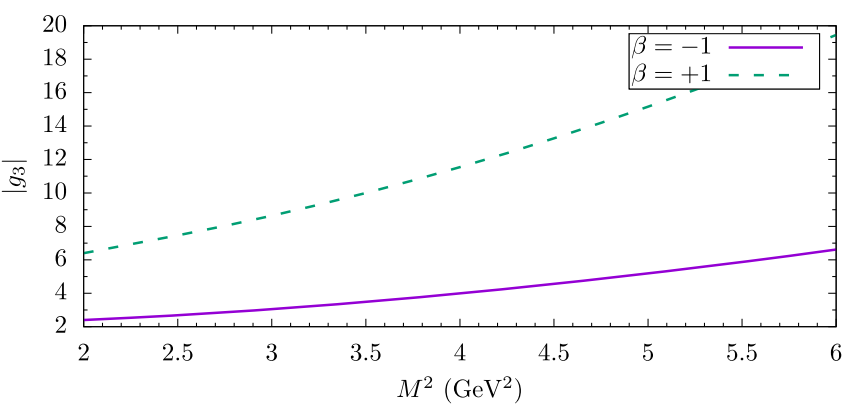

FIG. 4. The same as Fig. 3 but for $\left|g_{3}\right|$.

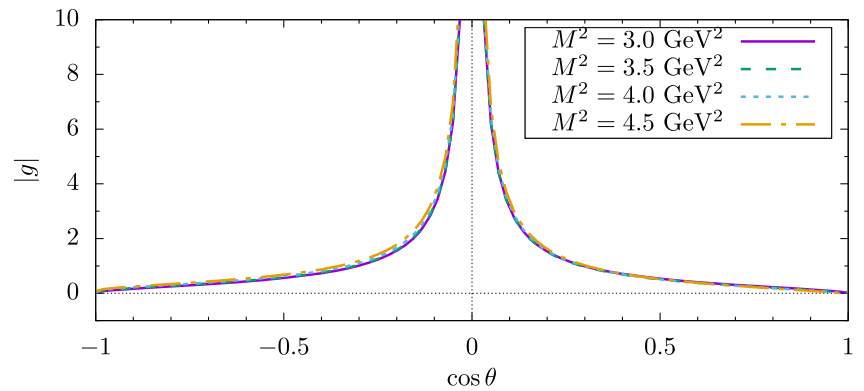

FIG. 5. The dependence of the coupling constant $|g|$ for the transition $\Xi_{c c}^{*++} \rightarrow \Xi_{c c}^{+} \pi^{+}$on $\cos \theta$ at shown values of $M^{2}$ and the fixed value of $s_{0}=18 \mathrm{GeV}^{2}$. 


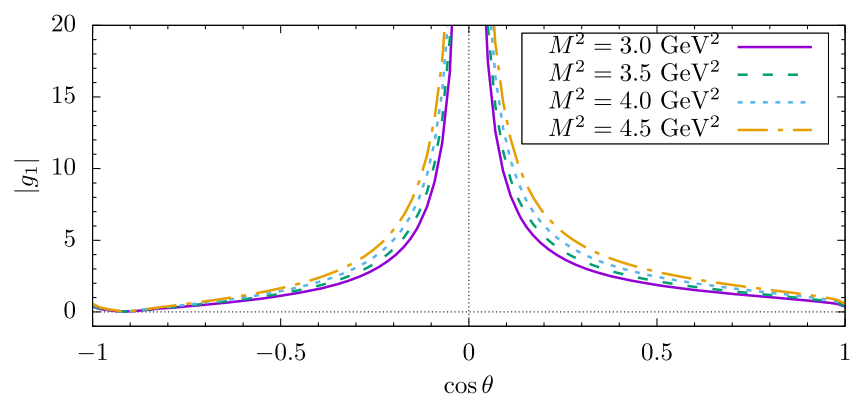

FIG. 6. The dependence of the coupling constant $\left|g_{1}\right|$ for the transition $\Xi_{c c}^{*++} \rightarrow \Xi_{c c}^{+} \rho^{+}$on $\cos \theta$ at shown values of $M^{2}$ and the fixed value of $s_{0}=18 \mathrm{GeV}^{2}$.

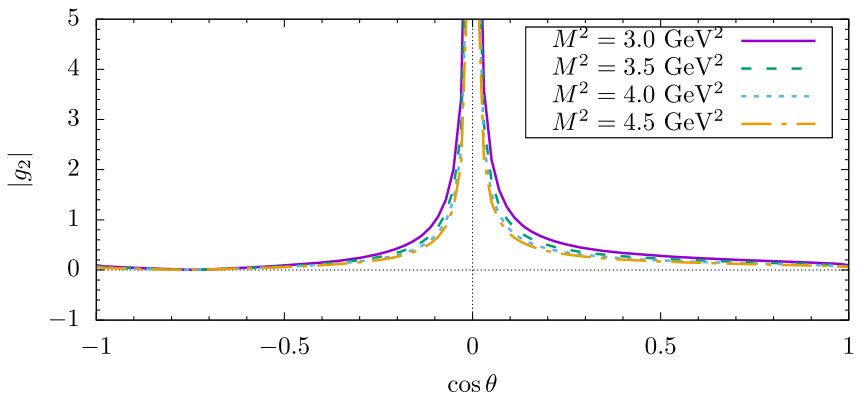

FIG. 7. The same as Fig. 6 but for $\left|g_{2}\right|$.

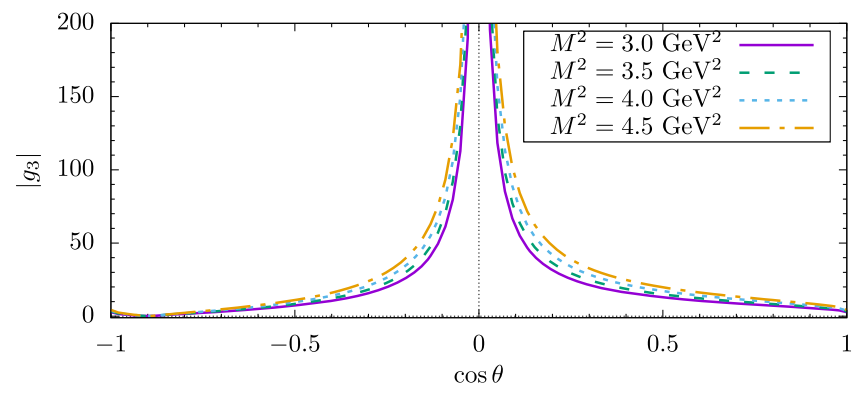

FIG. 8. The same as Fig. 6 but for $\left|g_{3}\right|$.

In addition to the input parameters summarized in Table I and the meson DAs, the LCSR involves three auxiliary parameters, i.e., the Borel mass parameter $M^{2}$, the continuum threshold $s_{0}$, and the arbitrary parameter $\beta$, which appears in the interpolating current. Hence, one should find the working regions of these parameters so that the LCSR is reliable. The lower bound of the Borel mass parameter can be obtained by insisting that the contributions from the highest-twist terms should be considerably smaller than the contributions from the lowest-twist terms. On the other
TABLE III. The numerical values of the moduli of the coupling constants $g$ of the light pseudoscalar mesons with the doubly heavy baryons.

\begin{tabular}{lcc}
\hline \hline Channel & General Current & Ioffe Current \\
\hline$\Xi_{c c}^{*++} \rightarrow \Xi_{c c}^{+} \pi^{+}$ & $0.39 \pm 0.02$ & $0.37 \pm 0.04$ \\
$\Xi_{b b}^{* 0} \rightarrow \Xi_{b b}^{-} \pi^{+}$ & $0.22 \pm 0.03$ & $0.20 \pm 0.04$ \\
$\Xi_{b c}^{*+} \rightarrow \Xi_{b c}^{0} \pi^{+}$ & $0.30 \pm 0.02$ & $0.26 \pm 0.3$ \\
$\Omega_{c c}^{*+} \rightarrow \Xi_{c c}^{+} K^{0}$ & $0.99 \pm 0.02$ & $0.78 \pm 0.08$ \\
$\Omega_{b b}^{*-} \rightarrow \Xi_{b b}^{-} K^{0}$ & $0.61 \pm 0.04$ & $0.43 \pm 0.07$ \\
$\Omega_{b c}^{* 0} \rightarrow \Xi_{b c}^{0} K^{0}$ & $0.78 \pm 0.03$ & $0.55 \pm 0.06$ \\
\hline \hline
\end{tabular}

hand, the upper limit of $M^{2}$ can be determined by demanding that the continuum contribution should not be too large. Meanwhile, the continuum threshold $s_{0}$ is obtained by requiring that the two-point sum rules reproduce a $10 \%$ accuracy of the mass of the doubly heavy baryons. These criteria lead to the values of $M^{2}$ and $s_{0}$ summarized in Table II for the transitions considered.

Our analysis reveals that the contributions from the twistfour terms in the considered domains of $M^{2}$ at the shown values of $s_{0}$ are smaller than $17 \%$ and higher states contribute $28 \%$ at maximum for all the considered channels. As an example, we present the $M^{2}$ dependence of $g$, $g_{1}, g_{2}$, and $g_{3}$ for $\Xi_{c c}^{*++} \rightarrow \Xi_{c c}^{+} \pi^{+}$and $\Xi_{c c}^{*++} \rightarrow \Xi_{c c}^{+} \rho^{+}$at fixed values of $s_{0}$ and $\beta$ in Figs. 1-4, respectively. Having determined the working regions of $M^{2}$ and $s_{0}$, one should find the working region of the auxiliary parameter, $\beta$. To do so, we investigate the dependence of $g, g_{1}, g_{2}$, and $g_{3}$ on $\cos \theta$, where $\theta$ is defined through the relation $\beta=\tan \theta$. As an illustration, we present the dependence of the said coupling constants for the transition $\Xi_{c c}^{*++} \rightarrow \Xi_{c c}^{+}$at fixed values of $M^{2}$ and $s_{0}$ in Figs. 5-8, respectively.

In Figs. 5-8, we observe that the coupling constants remain practically the same when $|\cos \theta|$ varies between 0.5 and 0.8. Our numerical analysis for the coupling constants of the doubly heavy baryons with the light mesons leads to the results presented in Tables III and IV. The uncertainties are due to the variation of the parameters $M^{2}, s_{0}$, and the errors in the values of the input parameters.

In Table III, we observe that the coupling constant $g$ of doubly heavy spin-3/2-spin-1/2 baryons with light pseudoscalar mesons are in good agreement with the ones for the case of the Ioffe current. In contrast, in Table IV, one can see that the coupling constants $g_{i}$ of the said baryons with light vector mesons differ drastically from the ones for the case of the Ioffe current.

Finally, we would like to note that the couplings for $\pi^{0}$ and $\rho^{0}$ mesons with doubly heavy baryons can be easily obtained from the obtained results by isospin symmetry. 
TABLE IV. The numerical values of the moduli of the coupling constants of the light vector mesons with the doubly heavy baryons.

\begin{tabular}{|c|c|c|c|c|c|c|}
\hline \multirow[b]{2}{*}{ Channel } & \multicolumn{3}{|c|}{ Case of the General Current } & \multicolumn{3}{|c|}{ Case of the Ioffe Current } \\
\hline & $\left|g_{1}\right|$ & $\left|g_{2}\right|$ & $\left|g_{3}\right|$ & $\left|g_{1}\right|$ & $\left|g_{2}\right|$ & $\left|g_{3}\right|$ \\
\hline$\Xi_{c c}^{*++} \rightarrow \Xi_{c c}^{+} \rho^{+}$ & $1.27 \pm 0.22$ & $0.10 \pm 0.02$ & $8.72 \pm 1.56$ & $0.59 \pm 0.10$ & $0.01 \pm 0.00$ & $3.71 \pm 0.64$ \\
\hline$\Xi_{b b}^{* 0} \rightarrow \Xi_{b b}^{-} \rho^{+}$ & $0.78 \pm 0.18$ & $0.02 \pm 0.00$ & $15.51 \pm 3.68$ & $0.33 \pm 0.08$ & $0.00 \pm 0.00$ & $6.47 \pm 1.52$ \\
\hline$\Xi_{b c}^{*+} \rightarrow \Xi_{b c}^{0} \rho^{+}$ & $1.08 \pm 0.16$ & $0.05 \pm 0.00$ & $14.60 \pm 2.24$ & $0.47 \pm 0.07$ & $0.00 \pm 0.00$ & $6.12 \pm 0.92$ \\
\hline$\Omega_{c c}^{*+} \rightarrow \Xi_{c c}^{+} K^{* 0}$ & $1.30 \pm 0.22$ & $0.33 \pm 0.06$ & $8.37 \pm 1.59$ & $0.59 \pm 0.10$ & $0.23 \pm 0.02$ & $3.10 \pm 0.57$ \\
\hline$\Omega_{b b}^{*-} \rightarrow \Xi_{b b}^{-} K^{* 0}$ & $0.85 \pm 0.19$ & $0.08 \pm 0.01$ & $16.50 \pm 3.90$ & $0.36 \pm 0.08$ & $0.05 \pm 0.00$ & $6.64 \pm 1.55$ \\
\hline$\Omega_{b c}^{* 0} \rightarrow \Xi_{b c}^{0} K^{* 0}$ & $1.12 \pm 0.17$ & $0.14 \pm 0.01$ & $14.61 \pm 2.32$ & $0.48 \pm 0.07$ & $0.09 \pm 0.00$ & $5.81 \pm 0.91$ \\
\hline
\end{tabular}

\section{CONCLUSION}

The discovery of $\Xi_{c c}^{+}$by the SELEX and $\Xi_{c c}^{++}$by the LHCb Collaborations stimulated theoretical and experimental studies for the investigation of the properties of other doubly heavy baryons in a new manner. Experimentally, the main attempt is focused on the discovery of doubly heavy baryons predicted by the quark model. Theoretically, the main effort is made to find promising decay channels, which can be potentially discovered in experiments. In this sense, one of the most important issues of doubly heavy baryons is the determination of the strong decay couplings among them. In the present work, we study the strong coupling constants of spin-3/2-to-spin- $1 / 2$ transitions with pseudoscalar ( $\pi$ and $K$ ) and vector $\left(\rho\right.$ and $\left.K^{*}\right)$ mesons. The obtained results on these strong coupling constants can carry useful information not only about the internal structure of doubly heavy baryons, but also about the nonperturbative interaction of these objects. The results on these coupling constants can play a useful role in deeper studies about the properties of doubly heavy baryons.

\section{APPENDIX A: DISTRIBUTION AMPLITUDES FOR LIGHT MESONS}

In this section, for completeness, we collect the matrix elements $\left\langle\mathcal{M}(q, \varepsilon)\left|\bar{q}(x) \Gamma_{i} q(0)\right| 0\right\rangle$ and $\langle\mathcal{M}(q, \varepsilon)| \bar{q}(x) \times$ $\Gamma_{i} G_{\mu \nu} q(0)|0\rangle$ and the relevant distribution amplitudes for light mesons together with the most recent values for the DA parameters involved [26-31].

\section{Pseudoscalar mesons}

Up to twist-four accuracy, the matrix elements $\langle\mathcal{P}(q, \varepsilon)|\bar{q}(x) \Gamma q(0)| 0\rangle$ and $\left\langle\mathcal{P}(q, \varepsilon)\left|\bar{q}(x) \Gamma G_{\mu \nu} q(0)\right| 0\right\rangle$ are given as follows:

$$
\begin{gathered}
\left\langle\mathcal{P}(p)\left|\bar{q}(x) \gamma_{\mu} \gamma_{5} q(0)\right| 0\right\rangle=-i f_{\mathcal{P}} p_{\mu} \int_{0}^{1} \mathrm{~d} u \mathrm{e}^{i \bar{u} p x}\left[\varphi_{\mathcal{P}}(u)+\frac{1}{16} m_{\mathcal{P}}^{2} x^{2} \hat{A}(u)\right]-\frac{i}{2} f_{\mathcal{P}} m_{\mathcal{P}}^{2} \frac{x_{\mu}}{p x} \int_{0}^{1} \mathrm{~d} u \mathrm{e}^{i \bar{u} p x} \hat{B}(u) \\
\left\langle\mathcal{P}(p)\left|\bar{q}(x) i \gamma_{5} q(0)\right| 0\right\rangle=\mu_{\mathcal{P}} \int_{0}^{1} \mathrm{~d} u \mathrm{e}^{i \bar{u} p x} \varphi_{P}(u) \\
\left\langle\mathcal{P}(p)\left|\bar{q}(x) \sigma_{\alpha \beta} \gamma_{5} q(0)\right| 0\right\rangle=\frac{i}{6} \mu_{\mathcal{P}}\left(1-\tilde{\mu}_{\mathcal{P}}^{2}\right)\left(p_{\alpha} x_{\beta}-p_{\beta} x_{\alpha}\right) \int_{0}^{1} \mathrm{~d} u \mathrm{e}^{i \bar{u} p x} \varphi_{\sigma}(u) \\
\left\langle\mathcal{P}(p)\left|\bar{q}(x) \sigma_{\mu \nu} \gamma_{5} g_{s} G_{\alpha \beta}(v x) q(0)\right| 0\right\rangle= \\
i \mu_{\mathcal{P}}\left\{p_{\alpha} p_{\mu}\left[g_{\nu \beta}-\frac{1}{p x}\left(p_{\nu} x_{\beta}+p_{\beta} x_{\nu}\right)\right]-p_{\alpha} p_{\nu}\left[g_{\mu \beta}-\frac{1}{p x}\left(p_{\mu} x_{\beta}+p_{\beta} x_{\mu}\right)\right]\right. \\
\left.-p_{\beta} p_{\mu}\left[g_{\nu \alpha}-\frac{1}{p x}\left(p_{\nu} x_{\alpha}+p_{\alpha} x_{\nu}\right)\right]+p_{\beta} p_{\nu}\left[g_{\mu \alpha}-\frac{1}{p x}\left(p_{\mu} x_{\alpha}+p_{\alpha} x_{\mu}\right)\right]\right\} \\
\times \int \mathcal{D} \alpha \mathrm{e}^{i\left(\alpha_{\bar{q}}+v \alpha_{g}\right) p x} \mathcal{T}\left(\alpha_{i}\right)
\end{gathered}
$$




$$
\begin{aligned}
\left\langle\mathcal{P}(p)\left|\bar{q}(x) \gamma_{\mu} \gamma_{5} g_{s} G_{\alpha \beta}(v x) q(0)\right| 0\right\rangle= & p_{\mu}\left(p_{\alpha} x_{\beta}-p_{\beta} x_{\alpha}\right) \frac{1}{p x} f_{\mathcal{P}} m_{\mathcal{P}}^{2} \int \mathcal{D} \alpha \mathrm{e}^{i\left(\alpha_{\bar{q}}+v \alpha_{g}\right) p x} \mathcal{A}_{\|}\left(\alpha_{i}\right) \\
& +\left\{p_{\beta}\left[g_{\mu \alpha}-\frac{1}{p x}\left(p_{\mu} x_{\alpha}+p_{\alpha} x_{\mu}\right)\right]-p_{\alpha}\left[g_{\mu \beta}-\frac{1}{p x}\left(p_{\mu} x_{\beta}+p_{\beta} x_{\mu}\right)\right]\right\} \\
& \times f_{\mathcal{P}} m_{\mathcal{P}}^{2} \int \mathcal{D} \alpha \mathrm{e}^{i\left(\alpha_{\bar{q}}+v \alpha_{g}\right) p x} \mathcal{A}_{\perp}\left(\alpha_{i}\right) \\
\left\langle\mathcal{P}(p)\left|\bar{q}(x) \gamma_{\mu} i g_{s} G_{\alpha \beta}(v x) q(0)\right| 0\right\rangle= & p_{\mu}\left(p_{\alpha} x_{\beta}-p_{\beta} x_{\alpha}\right) \frac{1}{p x} f_{\mathcal{P}} m_{\mathcal{P}}^{2} \int \mathcal{D} \alpha \mathrm{e}^{i\left(\alpha_{\bar{q}}+v \alpha_{g}\right) p x} V_{\|}\left(\alpha_{i}\right) \\
& +\left\{p_{\beta}\left[g_{\mu \alpha}-\frac{1}{p x}\left(p_{\mu} x_{\alpha}+p_{\alpha} x_{\mu}\right)\right]-p_{\alpha}\left[g_{\mu \beta}-\frac{1}{p x}\left(p_{\mu} x_{\beta}+p_{\beta} x_{\mu}\right)\right]\right\} \\
& \times f_{\mathcal{P}} m_{\mathcal{P}}^{2} \int \mathcal{D} \alpha \mathrm{e}^{i\left(\alpha_{\bar{q}}+v \alpha_{g}\right) p x} \mathcal{V}_{\perp}\left(\alpha_{i}\right)
\end{aligned}
$$

where

$$
\mu_{\mathcal{P}}=f_{\mathcal{P}} \frac{m_{\mathcal{P}}^{2}}{m_{q_{1}}+m_{q_{2}}}, \quad \tilde{\mu}_{\mathcal{P}}=\frac{m_{q_{1}}+m_{q_{2}}}{m_{\mathcal{P}}}
$$

where $m_{q_{1}}=m_{u}$ and $m_{q_{2}}=m_{d}$ for the pion, and $m_{q_{1}}=m_{u}$ and $m_{q_{2}}=m_{s}$ for the kaon. Here, $\varphi_{\mathcal{P}}(u), \hat{A}(u), \hat{B}(u), \varphi_{P}(u)$, $\varphi_{\sigma}(u), \mathcal{T}\left(\alpha_{i}\right), \mathcal{A}_{\perp}\left(\alpha_{i}\right), \mathcal{A}_{\|}\left(\alpha_{i}\right), \mathcal{V}_{\perp}\left(\alpha_{i}\right)$, and $\mathcal{V}_{\|}\left(\alpha_{i}\right)$ are the distribution amplitudes of the pseudoscalar meson with definite twist. The relevant DAs are as follows:

$$
\begin{gathered}
\phi_{\mathcal{P}}(u)=6 u \bar{u}\left[1+a_{1}^{\mathcal{P}} C_{1}(2 u-1)+a_{2}^{\mathcal{P}} C_{2}^{3 / 2}(2 u-1)\right] \\
\phi_{P}(u)=1+\left(30 \eta_{3}-\frac{5}{2} \frac{1}{\mu_{\mathcal{P}}^{2}}\right) C_{2}^{1 / 2}(2 u-1)+\left(-3 \eta_{3} w_{3}-\frac{27}{20} \frac{1}{\mu_{\mathcal{P}}^{2}}-\frac{81}{10} \frac{1}{\mu_{\mathcal{P}}^{2}} a_{2}^{\mathcal{P}}\right) C_{4}^{1 / 2}(2 u-1) \\
\phi_{\sigma}(u)=6 u \bar{u}\left[1+\left(5 \eta_{3}-\frac{1}{2} \eta_{3} w_{3}-\frac{7}{20} \mu_{\mathcal{P}}^{2}-\frac{3}{5} \mu_{\mathcal{P}}^{2} a_{2}^{\mathcal{P}}\right) C_{2}^{3 / 2}(2 u-1)\right] \\
\mathcal{T}\left(\alpha_{i}\right)=360 \eta_{3} a_{1} a_{2} a_{3}^{2}\left[1+w_{3} \frac{1}{2}\left(7 a_{3}-3\right)\right]
\end{gathered}
$$

where the $C_{n}^{k}(x)$ are the Gegenbauer polynomials and the values of the parameters inside the DAs at the renormalization scale of $\mu=1 \mathrm{GeV}$ are $a_{1}^{\pi}=0, a_{2}^{\pi}=0.44, a_{1}^{K}=0.06, a_{2}^{K}=0.25, \eta_{3}=0.015$, and $w_{3}=-3$ for the pion and $w_{3}=-1.2$ for the kaon. The accuracy of these input parameters are around $30 \%$.

\section{Vector mesons}

Up to twist-four accuracy, the matrix elements $\langle V(q, \varepsilon)|\bar{q}(x) \Gamma q(0)| 0\rangle$ and $\left\langle V(q, \varepsilon)\left|\bar{q}(x) \Gamma G_{\mu \nu} q(0)\right| 0\right\rangle$ are given as follows:

$$
\begin{aligned}
\left\langle V(q, \varepsilon)\left|\bar{q}_{1}(x) \gamma_{\mu} q_{2}(0)\right| 0\right\rangle= & f_{V} m_{V}\left(\frac{\varepsilon^{\lambda} \cdot x}{q \cdot x} q_{\mu} \int_{0}^{1} d u e^{i \bar{u} q \cdot x}\left(\phi_{2}^{\|}(u)+\frac{m_{V}^{2} x^{2}}{16} \phi_{4}^{\|}(u)\right)+\left(\varepsilon_{\mu}^{\lambda}-q_{\mu} \frac{\varepsilon^{\lambda} \cdot x}{q \cdot x}\right) \int_{0}^{1} d u e^{i \bar{u} q \cdot x} \phi_{3}^{\perp}(u)\right. \\
& \left.-\frac{1}{2} x_{\mu} \frac{\varepsilon^{\lambda} \cdot x}{(q \cdot x)^{2}} m_{V}^{2} \int_{0}^{1} d u e^{i \bar{u} q \cdot x}\left(\psi_{4}^{\|}(u)+\phi_{2}^{\|}(u)-2 \phi_{3}^{\perp}(u)\right)\right) \\
& \left\langle V(q, \varepsilon)\left|\bar{q}_{1}(x) \gamma_{\mu} \gamma_{5} q_{2}(0)\right| 0\right\rangle=-\frac{1}{4} \epsilon_{\mu}^{\nu \alpha \beta} \varepsilon_{\nu}^{\lambda} q_{\alpha} x_{\beta} f_{V} m_{V} \int_{0}^{1} d u \psi \frac{\perp}{3}(u)
\end{aligned}
$$




$$
\begin{aligned}
& \left\langle V(q, \varepsilon)\left|\bar{q}_{1}(x) \sigma_{\mu \nu} q_{2}(0)\right| 0\right\rangle=-i f_{V}^{T}\left(\left(\varepsilon_{\mu}^{\lambda} q_{\nu}-\varepsilon_{\nu}^{\lambda} q_{\mu}\right) \int_{0}^{1} d u e^{i \bar{u} q \cdot x} \phi_{2}^{\perp}(u)+\frac{m_{V}^{2} x^{2}}{16} \phi_{4}^{\perp}(u)\right. \\
& +\frac{\varepsilon \cdot x}{(q \cdot x)^{2}}\left(q_{\mu} x_{\nu}-q_{\nu} x_{\mu}\right) \int_{0}^{1} d u e^{i \bar{u} q \cdot x}\left(\phi_{3}^{\|}(u)-\frac{1}{2} \phi_{2}^{\perp}(u)-\frac{1}{2} \psi_{4}^{\perp}(u)\right) \\
& \left.+\frac{1}{2}\left(\varepsilon_{\mu}^{\lambda} x_{\nu}-\varepsilon_{\nu}^{\lambda} x_{\mu}\right) \frac{m_{V}^{2}}{q \cdot x} \int_{0}^{1} d u e^{i \bar{u} q \cdot x}\left(\psi_{4}^{\perp}(u)-\phi_{2}^{\perp}(u)\right)\right) \\
& \left\langle V(q, \varepsilon)\left|\bar{q}_{1}(x) \sigma_{\alpha \beta} g_{s} G_{\mu \nu}(u x) q_{2}(0)\right| 0\right\rangle=f_{V}^{T} m_{V}^{2} \frac{\varepsilon^{\lambda} \cdot x}{2 q \cdot x}\left(q_{\alpha} q_{\mu} g_{\beta \nu}^{\perp}-q_{\beta} q_{\mu} g_{\alpha \nu}^{\perp}-q_{\alpha} q_{\nu} g_{\beta \mu}^{\perp}+q_{\beta} q_{\nu} g_{\alpha \mu}^{\perp}\right) \int \mathcal{D} \alpha_{i} e^{i\left(\alpha_{1}+u \alpha_{3}\right) q \cdot x} \mathcal{T}\left(\alpha_{i}\right) \\
& +f_{V}^{T} m_{V}^{2}\left(q_{\alpha} \varepsilon_{\mu}^{\lambda} g_{\beta \nu}^{\perp}-q_{\alpha} \varepsilon_{\nu}^{\lambda} g_{\beta \mu}^{\perp}+q_{\beta} \varepsilon_{n}^{\lambda} u g_{\alpha \mu}^{\perp}\right) \int \mathcal{D} \alpha_{i} e^{i\left(\alpha_{1}+u \alpha_{3}\right) q \cdot x} \mathcal{T}_{1}^{(4)}\left(\alpha_{i}\right) \\
& +f_{V}^{T} m_{V}^{2}\left(q_{\mu} \varepsilon_{\alpha}^{\lambda} g_{\beta \nu}^{\perp}-q_{\mu} \varepsilon_{\beta}^{\lambda} g_{\alpha \nu}^{\perp}-q_{\nu} \varepsilon_{\alpha}^{\lambda} g_{\beta \mu}^{\perp}+q_{\nu} \varepsilon_{\beta}^{\lambda} g_{\alpha \mu}^{\perp}\right) \int \mathcal{D} \alpha_{i} e^{i\left(\alpha_{1}+u \alpha_{3}\right) q \cdot x} \mathcal{T}_{2}^{(4)}\left(\alpha_{i}\right) \\
& +\frac{f_{V}^{T} m_{V}^{2}}{q \cdot x}\left(q_{\alpha} q_{\mu} \varepsilon_{\beta}^{\lambda} x_{\nu}-q_{\beta} q_{\mu} \varepsilon_{\alpha}^{\lambda} x_{\nu}-q_{\alpha} q_{\nu} \varepsilon_{\beta}^{\lambda} x_{\mu}+q_{\beta} q_{\nu} \varepsilon_{\alpha}^{\lambda} x_{\mu}\right) \int \mathcal{D} \alpha_{i} e^{i\left(\alpha_{1}+u \alpha_{3}\right) q \cdot x} \mathcal{T}_{3}^{(4)}\left(\alpha_{i}\right) \\
& +\frac{f_{V}^{T} m_{V}^{2}}{q \cdot x}\left(q_{\alpha} q_{\mu} \varepsilon_{\nu}^{\lambda} x_{\beta}-q_{\beta} q_{\mu} \varepsilon_{\nu}^{\lambda} x_{\alpha}-q_{\alpha} q_{\nu} \varepsilon_{\mu}^{\lambda} x_{\beta}+q_{\beta} q_{\nu} \varepsilon_{\mu}^{\lambda} x_{\alpha}\right) \int \mathcal{D} \alpha_{i} e^{i\left(\alpha_{1}+u \alpha_{3}\right) q \cdot x} \mathcal{T}_{4}^{(4)}\left(\alpha_{i}\right) \\
& \left\langle V(q, \varepsilon)\left|\bar{q}_{1}(x) g_{s} G_{\mu \nu}(u x) q_{2}(0)\right| 0\right\rangle=-i f_{V}^{T} m_{V}\left(\varepsilon_{\mu}^{\lambda} q_{\nu}-\varepsilon_{\nu}^{\lambda} q_{\mu}\right) \int \mathcal{D} \alpha_{i} e^{i\left(\alpha_{1}+u \alpha_{3}\right) q \cdot x} \mathcal{S}\left(\alpha_{i}\right) \\
& \left\langle V(q, \lambda)\left|\bar{q}_{1}(x) g_{s} \tilde{G}_{\mu \nu}(u x) \gamma_{5} q_{2}(0)\right| 0\right\rangle=-i f_{V}^{T} m_{V}\left(\varepsilon_{\mu}^{\lambda} q_{\nu}-\varepsilon_{\nu}^{\lambda} q_{\mu}\right) \int \mathcal{D} \alpha_{i} e^{i\left(\alpha_{1}+u \alpha_{3}\right) q \cdot x} \tilde{\mathcal{S}}\left(\alpha_{i}\right) \\
& \left\langle V(q, \varepsilon)\left|\bar{q}_{1}(x) g_{s} \tilde{G}_{\mu \nu}(u x) \gamma_{\alpha} \gamma_{5} q_{2}(0)\right| 0\right\rangle=f_{V} m_{V} q_{\alpha}\left(\varepsilon_{\mu}^{\lambda} q_{\nu}-\varepsilon_{n}^{\lambda} u q_{\mu}\right) \int \mathcal{D} \alpha_{i} e^{i\left(\alpha_{1}+u \alpha_{3}\right) q \cdot x} \mathcal{A}\left(\alpha_{i}\right) \\
& \left\langle V(q, \varepsilon)\left|\bar{q}_{1}(x) g_{s} G_{\mu \nu}(u x) i \gamma_{\alpha} q_{2}(0)\right| 0\right\rangle=f_{V} m_{V} q_{\alpha}\left(\varepsilon_{\mu}^{\lambda} q_{\nu}-\varepsilon_{\nu}^{\lambda} q_{\mu}\right) \int \mathcal{D} \alpha_{i} e^{i\left(\alpha_{1}+u \alpha_{3}\right) q \cdot x} \mathcal{V}\left(\alpha_{i}\right)
\end{aligned}
$$

where $\tilde{G}_{\mu \nu}=\frac{1}{2} \epsilon_{\mu \nu \alpha \beta} G^{\alpha \beta}$ is the dual gluon field strength tensor and $\int \mathcal{D} \alpha_{i}=\int d \alpha_{1} d \alpha_{2} d \alpha_{3} \delta\left(1-\alpha_{1}-\alpha_{2}-\alpha_{3}\right)$. Now we list the DAs.

\section{Two-particle twist-two DAs}

$$
\begin{gathered}
\phi_{2}^{\|}(u)=6 \bar{u}\left(1+a_{1}^{\|} C_{1}^{3 / 2}(\xi)+a_{2}^{\|} C_{2}^{3 / 2}(\xi)\right) u \\
\phi_{2}^{\perp}(u)=6 \bar{u}\left(1+a_{1}^{\perp} C_{1}^{3 / 2}(\xi)+a_{2}^{\perp} C_{2}^{3 / 2}(\xi)\right) u
\end{gathered}
$$

\section{Two-particle twist-three DAs}

$$
\begin{aligned}
\phi_{3}^{\|}(u)= & 3 \xi^{2}+\left(3 a_{1}^{\perp} \xi\left(-1+3 \xi^{2}\right)\right) / 2+\left(\left(15 \kappa_{3}^{\perp}\right) / 2-\left(3 \lambda \frac{\perp}{3}\right) / 4\right) \xi\left(-3+5 \xi^{2}\right) \\
& +\left(3 a_{2}^{\perp} \xi^{2}\left(-3+5 \xi^{2}\right)\right) / 2+\left(5 \omega_{3}^{\perp}\left(3-30 \xi^{2}+35 \xi^{4}\right)\right) / 8-\left(3 f _ { V } ( m _ { q _ { 1 } } - m _ { q _ { 2 } } ) \xi \left(2+9 a_{1}^{\|} \xi \times 2 a_{2}^{\|}(11-30 \bar{u} u)\right.\right. \\
& \left.\left.+\left(1+6 a_{2}^{\|}+3 a_{1}^{\|}\right) \ln (\bar{u})+\left(1+6 a_{2}^{\|}-3 a_{1}^{\|}\right) \ln (u)\right)\right) /\left(2 f_{V}^{T} m_{V}\right)+\left(3 f _ { V } ( m _ { q _ { 1 } } + m _ { q _ { 2 } } ) \left(1+8 a_{1}^{\|} \xi+3 a_{2}^{\|}(7-30 \bar{u} u)\right.\right. \\
& \left.\left.+\left(1+6 a_{2}^{\|}+3 a_{1}^{\|}\right) \xi \ln (\bar{u})-\left(1+6 a_{2}^{\|}-3 a_{1}^{\|}\right) \xi \ln (u)\right)\right) /\left(2 f_{V}^{T} m_{V}\right)
\end{aligned}
$$




$$
\begin{aligned}
& \psi_{3}^{\|}(u)= 6 \bar{u}\left(1+C_{1}^{3 / 2}(\xi)\left(a_{1}^{\perp} / 3+\left(5 \kappa_{3}^{\perp}\right) / 3\right)-\left(C_{3}^{3 / 2}(\xi) \lambda_{3}^{\perp}\right) / 20+C_{2}^{3 / 2}(\xi)\left(a_{2}^{\perp} / 6+\left(5 \omega_{3}^{\perp}\right) / 18\right)\right) \\
& \times u-\left(3 f _ { V } ( m _ { q _ { 1 } } - m _ { q _ { 2 } } ) \left(\bar{u}\left(9 a_{1}^{\|}+10 a_{2}^{\|} \xi\right) u+\left(1+6 a_{2}^{\|}+3 a_{1}^{\|}\right) \bar{u} \ln (\bar{u})-\left(1+6 a_{2}^{\|}-3 a_{1}^{\|}\right)\right.\right. \\
&\times u \ln (u))) /\left(f_{V}^{T} m_{V}\right)+\left(3 f _ { V } ( m _ { q _ { 1 } } + m _ { q _ { 2 } } ) \left(\bar{u} u\left(1+2 a_{1}^{\|} \xi+3 a_{2}^{\|}(7-5 \bar{u} u)\right)+\left(1+6 a_{2}^{\|}+3 a_{1}^{\|}\right) \bar{u} \ln (\bar{u})\right.\right. \\
&\left.\left.+\left(1+6 a_{2}^{\|}-3 a_{1}^{\|}\right) u \ln (u)\right)\right) /\left(f_{V}^{T} m_{V}\right) \\
& \psi_{3}^{\perp}(u)=6 \bar{u}\left(1+C_{1}^{3 / 2}(\xi)\left(a_{1}^{\|} / 3+\left(20 \kappa_{3}^{\|}\right) / 9\right)+C_{3}^{3 / 2}(\xi)\left(-\lambda_{3}^{\|} / 8+\tilde{\lambda}_{3}^{\|} / 4\right)+C_{2}^{3 / 2}(\xi)\left(a_{2}^{\|} / 6+\left(5 \omega_{3}^{\|}\right) / 12-\left(5 \tilde{\omega}_{3}^{\|}\right) / 24\right.\right. \\
&\left.\left.+\left(10 \zeta_{3}^{\|}\right) / 9\right)\right) u-\left(6 f _ { V } ^ { T } ( m _ { q _ { 1 } } - m _ { q _ { 2 } } ) \left(\bar{u}\left(9 a_{1}^{\perp}+10 a_{2}^{\perp} \xi\right) u+\left(1+6 a_{2}^{\perp}+3 a_{1}^{\perp}\right) \bar{u} \ln (\bar{u})\right.\right. \\
&\left.\left.-\left(1+6 a_{2}^{\perp}-3 a_{1}^{\perp}\right) u \ln (u)\right)\right) /\left(f_{V} m_{V}\right)+\left(6 f _ { V } ^ { T } ( m _ { q _ { 1 } } + m _ { q _ { 2 } } ) \left(\bar{u} u \times\left(2+3 a_{1}^{\perp} \xi+2 a_{2}^{\perp}(11-10 \bar{u} u)\right)\right.\right. \\
&\left.\left.+\left(1+6 a_{2}^{\perp}+3 a_{1}^{\perp}\right) \bar{u} \ln (\bar{u})+\left(1+6 a_{2}^{\perp}-3 a_{1}^{\perp}\right) \times u \ln (u)\right)\right) /\left(f_{V} m_{V}\right) \\
& \phi_{3}^{\perp}(u)=\left(3 a_{1}^{\|} \xi^{3}\right) / 2+\left(3\left(1+\xi^{2}\right)\right) / 4+\left(5 \kappa_{3}^{\|}-\left(15 \lambda_{3}^{\|}\right) / 16+\left(15 \tilde{\lambda}_{3}^{\|}\right) / 8\right) \xi\left(-3+5 \xi^{2}\right) \\
&+\left(\left(9 a_{2}^{\|}\right) / 112+\left(15 \omega_{3}^{\|}\right) / 32-\left(15 \tilde{\omega}_{3}^{\|}\right) / 64\right)\left(3-30 \xi^{2}+35 \xi^{4}\right)+\left(-1+3 \xi^{2}\right)\left(\left(3 a_{2}^{\|}\right) / 7\right. \\
&\left.+5 \zeta_{3}^{\|}\right)-\left(3 f _ { V } ^ { T } ( m _ { q _ { 1 } } - m _ { q _ { 2 } } ) \left(2 \xi+2 a_{2}^{\perp} \xi(11-20 \bar{u} u)+9 a_{1}^{\perp}(1-2 \bar{u} u)+\left(1+6 a_{2}^{\perp}+3 a_{1}^{\perp}\right) \ln (\bar{u})\right.\right. \\
&\left.\left.-\left(1+6 a_{2}^{\perp}-3 a_{1}^{\perp}\right) \ln (u)\right)\right) /\left(2 f_{V} m_{V}\right)+\left(3 f _ { V } ^ { T } ( m _ { q _ { 1 } } + m _ { q _ { 2 } } ) \left(2+9 a_{1}^{\perp} \xi+2 a_{2}^{\perp}(11-30 \bar{u} u)\right.\right. \\
&\left.\left.+\left(1+6 a_{2}^{\perp}+3 a_{1}^{\perp}\right) \ln (\bar{u})+\left(1+6 a_{2}^{\perp}-3 a_{1}^{\perp}\right) \ln (u)\right)\right) /\left(2 f_{V} m_{V}\right) .
\end{aligned}
$$

\section{Two-particle twist-four DAs}

$$
\begin{aligned}
\psi_{4}^{\|}(u)= & +C_{3}^{1 / 2}(\xi)\left(\left(-9 a_{1}^{\|}\right) / 5-\left(20 \kappa_{3}^{\|}\right) / 3-\left(16 \kappa_{4}^{\|}\right) / 3\right)+C_{1}^{1 / 2}(\xi)\left(\left(9 a_{1}^{\|}\right) / 5+12 \kappa_{4}^{\|}\right) \\
& +C_{3}^{1 / 2}(\xi)\left(-5 \theta_{2}^{\|}+10 \theta_{1}^{\|}\right)+\left(6 f _ { V } ^ { T } ( m _ { q _ { 1 } } - m _ { q _ { 2 } } ) \left(\xi+\left(a_{1}^{\perp}\left(-1+3 \xi^{2}\right)\right) / 2\right.\right. \\
& +\left(5 \kappa_{3}^{\perp}\left(-1+3 \xi^{2}\right)\right) / 2+\left(a_{2}^{\perp} \xi\left(-3+5 \xi^{2}\right)\right) / 2+\left(5 \omega_{3}^{\perp} \xi\left(-3+5 \xi^{2}\right)\right) / 6 \\
& \left.\left.-\left(\lambda \frac{\perp}{3}\left(3-30 \xi^{2}+35 \xi^{4}\right)\right) / 16\right)\right) /\left(f_{V} m_{V}\right)+C_{4}^{1 / 2}(\xi)\left(\left(-27 a_{2}^{\|}\right) / 28-\left(15 \omega_{3}^{\|}\right) / 8\right. \\
& \left.-\left(15 \tilde{\omega}_{3}^{\|}\right) / 16+\left(5 \zeta_{3}^{\|}\right) / 4\right)+C_{2}^{1 / 2}(\xi)\left(-1-\left(2 a_{2}^{\|}\right) / 7+\left(40 \xi_{3}^{\|}\right) / 3\right)-\left(20 C_{2}^{1 / 2}(\xi) \zeta_{4}^{\|}\right) / 3 \\
\phi_{4}^{\|}(u)= & \left(6 \overline { u } f _ { V } ^ { T } ( m _ { q _ { 1 } } - m _ { q _ { 2 } } ) \left(-\left(C_{1}^{3 / 2}(\xi)\left(\left(82 a_{1}^{\perp}\right) / 5+10 \kappa_{3}^{\perp}\right)\right)+C_{3}^{3 / 2}(\xi)\left(\left(2 a_{1}^{\perp}\right) / 5+\left(7 \lambda_{3}^{\perp}\right) / 54\right)\right.\right. \\
+ & \left(2 C_{5}^{3 / 2}(\xi) \lambda \frac{\perp}{3}\right) / 135+C_{4}^{3 / 2}(\xi)\left(-2 / 315+a_{2}^{\perp} / 5-\omega_{3}^{\perp} / 21\right)+20 C_{2}^{3 / 2}(\xi)(10 / 189 \\
+ & \left.\left.\left.a_{2}^{\perp} / 3-\omega_{3}^{\perp} / 21\right)\right) u\right) /\left(f_{V} m_{V}\right)+\left(6 \overline { u } f _ { V } ^ { T } ( m _ { q _ { 1 } } + m _ { q _ { 2 } } ) \left(2\left(3+16 a_{2}^{\perp}\right)+\left(1 0 C _ { 1 } ^ { 3 / 2 } ( \xi ) \left(-a_{1}^{\perp}\right.\right.\right.\right. \\
+ & \left.\left.\left.\left.\kappa_{3}^{\perp}\right)\right) / 3-\left(C_{3}^{3 / 2}(\xi) \lambda \frac{\perp}{\perp}\right) / 10+C_{2}^{3 / 2}(\xi)\left(-a_{2}^{\perp}+\left(5 \omega_{3}^{\perp}\right) / 9\right)\right) u\right) /\left(f_{V} m_{V}\right) \\
+ & 30 \bar{u}^{2}\left(C_{1}^{5 / 2}(\xi)\left(\left(17 a_{1}^{\|}\right) / 50-\lambda_{3}^{\|} / 5+\left(2 \tilde{\lambda}_{3}^{\|}\right) / 5\right)+\left(C _ { 2 } ^ { 5 / 2 } ( \xi ) \left(\left(9 a_{2}^{\|}\right) / 7+\left(7 \omega_{3}^{\|}\right) / 6\right.\right.\right. \\
- & \left.\left.\left.\left(3 \tilde{\omega}_{3}^{\|}\right) / 4+\zeta_{3}^{\|} / 9\right)\right) / 10+\left(4\left(1+a_{2}^{\|} / 21+\left(10 \zeta_{3}^{\|}\right) / 9\right)\right) / 5\right) u^{2}+30 \bar{u}^{2}\left(C _ { 1 } ^ { 5 / 2 } ( \xi ) \left(\left(2 \theta_{2}^{\|}\right) / 3\right.\right. \\
- & \left.\left.\left(8 \theta_{1}^{\|}\right) / 15\right)+\left(20 \zeta_{4}^{\|}\right) / 9\right) u^{2}+\left(f _ { V } ^ { T } ( m _ { q _ { 1 } } - m _ { q _ { 2 } } ) \left(\left(-23-108 a_{2}^{\perp}-54 a_{1}^{\perp}+5 u^{2}\right) \ln (\bar{u})\right.\right. \\
& \left.\left.-\left(-23-108 a_{2}^{\perp}+54 a_{1}^{\perp}+5 \bar{u}^{2}\right) \ln (u)\right)\right) /\left(f_{V} m_{V}\right)+\left(2 4 f _ { V } ^ { T } ( m _ { q _ { 1 } } + m _ { q _ { 2 } } ) \left(\left(1+6 a_{2}^{\perp}\right.\right.\right. \\
+ & \left.\left.\left.3 a_{1}^{\perp}\right) \bar{u}^{2} \ln (\bar{u})+\left(1+6 a_{2}^{\perp}-3 a_{1}^{\perp}\right) u^{2} \ln (u)\right)\right) /\left(f_{V} m_{V}\right)+4\left(a_{1}^{\|}-\left(40 \kappa_{3}^{\|}\right) / 3\right)((11 \\
& \left.\left.-3 \xi^{2}\right) / 8-(2-\bar{u}) \bar{u}^{3} \ln (\bar{u})+(2-u) u^{3} \ln (u)\right)+80 \psi_{2}^{\|}\left(\left(11-3 \xi^{2}\right) / 8\right. \\
& \left.-(2-\bar{u}) \bar{u}^{3} \ln (\bar{u})+(2-u) u^{3} \ln (u)\right)-80 \tilde{\omega}_{4}^{\|}\left(\left(\bar{u}\left(21-13 \xi^{2}\right) u\right) / 8+\bar{u}^{3}(10-15 \bar{u}\right. \\
+ & \left.\left.6 \bar{u}^{2}\right) \ln (\bar{u})+u^{3}\left(10-15 u+6 u^{2}\right) \ln (u)\right)+2\left(-2 a_{2}^{\|}+3 \omega_{3}^{\|}-\left(14 \zeta_{3}^{\|}\right) / 3\right)((\bar{u}(21 \\
- & \left.\left.\left.13 \xi^{2}\right) u\right) / 8+\bar{u}^{3}\left(10-15 \bar{u}+6 \bar{u}^{2}\right) \ln (\bar{u})+u^{3}\left(10-15 u+6 u^{2}\right) \ln (u)\right)
\end{aligned}
$$




$$
\begin{aligned}
\psi_{4}^{\perp}(u)= & 1+C_{1}^{1 / 2}(\xi)\left(\left(-3 a_{1}^{\perp}\right) / 5+12 \kappa_{4}^{\perp}\right)+\left(C_{5}^{1 / 2}(\xi) \lambda \frac{\perp}{\perp}\right) / 3+C_{4}^{1 / 2}(\xi)\left(\left(-3 a_{2}^{\perp}\right) / 7\right. \\
& \left.-\left(5 \omega_{3}^{\perp}\right) / 4\right)+C_{3}^{1 / 2}(\xi)\left(\left(3 a_{1}^{\perp}\right) / 5-5 \kappa_{3}^{\perp}-12 \kappa_{4}^{\perp}-\lambda \frac{\perp}{3} / 3+5\left(\left(-\theta_{2}^{\perp}-\tilde{\theta}_{2}^{\perp}\right) / 2+\theta_{1}^{\perp}\right.\right. \\
& \left.\left.+\tilde{\theta}_{1}^{\perp}\right)\right)+\left(f _ { V } ( m _ { q _ { 1 } } + m _ { q _ { 2 } } ) \left(3\left(1+6 a_{2}^{\|}\right)+3 a_{1}^{\|} C_{1}^{1 / 2}(\xi)+5 C_{3}^{1 / 2}(\xi)\left(4 \kappa_{3}^{\|}-\left(3 \lambda_{3}^{\|}\right) / 4\right.\right.\right. \\
& \left.\left.\left.+\left(3 \tilde{\lambda}_{3}^{\|}\right) / 2\right)+\left(15 C_{4}^{1 / 2}(\xi)\left(2 \omega_{3}^{\|}-\tilde{\omega}_{3}^{\|}\right)\right) / 4+5 C_{2}^{1 / 2}(\xi)\left(-3 a_{2}^{\|}+4 \zeta_{3}^{\|}\right)\right)\right) /\left(f_{V}^{T} m_{V}\right) \\
& +C_{2}^{1 / 2}(\xi)\left(-1+\left(3 a_{2}^{\perp}\right) / 7-10\left(\zeta_{4}^{\perp}+\tilde{\zeta}_{4}^{\perp}\right)\right)-\left(6 \bar{u} f_{V}\left(m_{q_{1}}-m_{q_{2}}\right)\left(9 a_{1}^{\|}+10 a_{2}^{\|} \xi\right) \times u\right) /\left(f_{V}^{T} m_{V}\right) \\
& +\left(6 f_{V}\left(m_{q_{1}}-m_{q_{2}}\right)\left(-\left(\left(1+6 a_{2}^{\|}+3 a_{1}^{\|}\right) \bar{u} \ln (\bar{u})\right)+\left(1+6 a_{2}^{\|}-3 a_{1}^{\|}\right) \times u \ln (u)\right)\right) /\left(f_{V}^{T} m_{V}\right) \\
& +\left(6 f_{V}\left(m_{q_{1}}+m_{q_{2}}\right)\left(\left(1+6 a_{2}^{\|}+3 a_{1}^{\|}\right) \bar{u} \ln (\bar{u})+\left(1+6 a_{2}^{\|}-3 a_{1}^{\|}\right) \times u \ln (u)\right)\right) /\left(f_{V}^{T} m_{V}\right) \\
\phi_{4}^{\perp}(u)= & 30 \bar{u}^{2}\left(2 / 5+\left(4 a_{2}^{\perp}\right) / 35-\left(4 C_{3}^{5 / 2}(\xi) \lambda \frac{\perp}{3}\right) / 1575+C_{2}^{5 / 2}(\xi)\left(\left(3 a_{2}^{\perp}\right) / 35+\omega_{3}^{\perp} / 60\right)\right. \\
& +C_{1}^{5 / 2}(\xi)\left(\left(3 a_{1}^{\perp}\right) / 25+\kappa_{3}^{\perp} / 3-\lambda \frac{1}{3} / 45+\left(7 \theta_{2}^{\perp}\right) / 30-\left(3 \tilde{\theta}_{2}^{\perp}\right) / 20-\theta_{1}^{\perp} / 15+\tilde{\theta}_{1}^{\perp} / 5\right) \\
& \left.+\left(4 \zeta_{4}^{\perp}\right) / 3-\left(8 \tilde{\zeta}_{4}^{\perp}\right) / 3\right) u^{2}+\left(-a_{1}^{\perp}+5 \kappa_{3}^{\perp}-20 \tilde{\phi}_{2}^{\perp}\right)\left(\left(\bar{u} \xi\left(-11+3 \xi^{2}\right) u\right) / 2+4(2-\bar{u})\right. \\
& \left.\quad \times \bar{u}^{3} \ln (\bar{u})-4(2-u) u^{3} \ln (u)\right)+\left(\left(-36 a_{2}^{\perp}\right) / 11-\left(252\left\langle\left\langle Q^{(1)}\right\rangle\right) / 55-\left(140\left\langle\left\langle Q^{(3)}\right\rangle\right) / 11\right.\right.\right. \\
& \left.+2 \omega_{3}^{\perp}\right)\left(-\left(\bar{u}\left(-21+13 \xi^{2}\right) u\right) / 8+\bar{u}^{3}\left(10-15 \bar{u}+6 \bar{u}^{2}\right) \ln (\bar{u})+u^{3}\left(10-15 u+6 u^{2}\right) \ln (u)\right) .
\end{aligned}
$$

\section{Three-particle twist-three DAs}

$$
\begin{gathered}
\mathcal{S}\left(\alpha_{1}, \alpha_{3}\right)=30 \alpha_{3}^{2}\left(\left(\left(-3\left(\alpha_{1}^{2}+\left(1-\alpha_{1}-\alpha_{3}\right)^{2}\right)\right) / 2+\left(1-\alpha_{3}\right) \alpha_{3}\right) \psi_{2}^{\perp}+\left(-6 \alpha_{1}\left(1-\alpha_{1}-\alpha_{3}\right)\right.\right. \\
\left.\left.+\left(1-\alpha_{3}\right) \alpha_{3}\right) \psi_{1}^{\perp}+\left(1-\alpha_{3}\right) \psi_{0}^{\perp}-\left(-1+2 \alpha_{1}+\alpha_{3}\right)\left(\left(\left(-3+5 \alpha_{3}\right) \theta_{2}^{\perp}\right) / 2+\alpha_{3} \theta_{1}^{\perp} \theta_{0}^{\perp}\right)\right) \\
\tilde{\mathcal{S}}\left(\alpha_{1}, \alpha_{3}\right)=30 \alpha_{3}^{2}\left(\left(\left(-3\left(\alpha_{1}^{2}+\left(1-\alpha_{1}-\alpha_{3}\right)^{2}\right)\right) / 2+\left(1-\alpha_{3}\right) \alpha_{3}\right) \tilde{\psi}_{2}^{\perp}+\left(-6 \alpha_{1}\left(1-\alpha_{1}-\alpha_{3}\right)\right.\right. \\
\left.\left.+\left(1-\alpha_{3}\right) \alpha_{3}\right) \tilde{\psi}_{1}^{\perp}+\left(1-\alpha_{3}\right) \tilde{\psi}_{0}^{\perp}-\left(\alpha_{1}-\alpha_{3}\right)\left(\left(\left(-3+5 \alpha_{3}\right) \tilde{\theta}_{2}^{\perp}\right) / 2+\alpha_{3} \tilde{\theta}_{1}^{\perp} \tilde{\theta}_{0}^{\perp}\right)\right) \\
\mathcal{V}\left(\alpha_{1}, \alpha_{3}\right)=360 \alpha_{1}\left(1-\alpha_{1}-\alpha_{3}\right) \alpha_{3}^{2}\left(\kappa_{3}^{\|}+\left(\left(-3+7 \alpha_{3}\right) \lambda_{3}^{\|}\right) / 2+\left(-1+2 \alpha_{1}+\alpha_{3}\right) \omega_{3}^{\|}\right) \\
\mathcal{A}\left(\alpha_{1}, \alpha_{3}\right)=360 \alpha_{1}\left(1-\alpha_{1}-\alpha_{3}\right) \alpha_{3}^{2}\left(\left(-1+2 \alpha_{1}+\alpha_{3}\right) \tilde{\lambda}_{3}^{\|}+\left(\left(-3+7 \alpha_{3}\right) \tilde{\omega}_{3}^{\|}\right) / 2+\zeta_{3}^{\|}\right) \\
\mathcal{T}\left(\alpha_{1}, \alpha_{3}\right)=360 \alpha_{1}\left(1-\alpha_{1}-\alpha_{3}\right) \alpha_{3}^{2}\left(\kappa_{3}^{\perp}+\left(\left(-3+7 \alpha_{3}\right) \lambda_{3}^{\perp}\right) / 2+\left(-1+2 \alpha_{1}+\alpha_{3}\right) \omega_{3}^{\perp}\right) .
\end{gathered}
$$

\section{Three-particle twist-four DAs}

$$
\begin{gathered}
\mathcal{T}_{1}^{(4)}\left(\alpha_{1}, \alpha_{3}\right)=120 \alpha_{1}\left(1-\alpha_{1}-\alpha_{3}\right) \alpha_{3}\left(\left(-1+2 \alpha_{1}+\alpha_{3}\right) \phi_{1}^{\perp}+\phi_{0}^{\perp}+\left(-1+3 \alpha_{3}\right) \phi_{2}^{\perp}\right) \\
\mathcal{T}_{2}^{(4)}\left(\alpha_{1}, \alpha_{3}\right)=-30 \alpha_{3}^{2}\left(-\left(\left(-1+2 \alpha_{1}+\alpha_{3}\right)\left(\left(\left(-3+5 \alpha_{3}\right) \tilde{\psi}_{2}^{\perp}\right) / 2+\alpha_{3} \tilde{\psi}_{1}^{\perp}+\tilde{\psi}_{0}^{\perp}\right)\right)+\left(\left(-3\left(\alpha_{1}^{2}+\left(1-\alpha_{1}-\alpha_{3}\right)^{2}\right)\right) / 2\right.\right. \\
\left.\left.+\left(1-\alpha_{3}\right) \alpha_{3}\right) \tilde{\theta}_{2}^{\perp}+\left(-6 \alpha_{1}\left(1-\alpha_{1}-\alpha_{3}\right)+\left(1-\alpha_{3}\right) \alpha_{3}\right) \tilde{\theta}_{1}^{\perp}+\left(1-\alpha_{3}\right) \tilde{\theta}_{0}^{\perp}\right) \\
\mathcal{T}_{3}^{(4)}\left(\alpha_{1}, \alpha_{3}\right)=-120 \alpha_{1}\left(1-\alpha_{1}-\alpha_{3}\right) \alpha_{3}\left(\left(-1+3 \alpha_{3}\right) \tilde{\phi}_{2}^{\perp}+\left(-1+2 \alpha_{1}+\alpha_{3}\right) \tilde{\phi}_{1}^{\perp}+\tilde{\phi}_{0}^{\perp}\right) \\
\mathcal{T}_{4}^{(4)}\left(\alpha_{1}, \alpha_{3}\right)=30 \alpha_{3}^{2}\left(-\left(\left(-1+2 \alpha_{1}+\alpha_{3}\right)\left(\left(\left(-3+5 \alpha_{3}\right) \psi \frac{\perp}{\perp}\right) / 2+\alpha_{3} \psi_{1}^{\perp}+\psi_{0}^{\perp}\right)\right)+\left(\left(-3\left(\alpha_{1}^{2}+\left(1-\alpha_{1}-\alpha_{3}\right)^{2}\right)\right) / 2\right.\right. \\
\left.\left.+\left(1-\alpha_{3}\right) \alpha_{3}\right) \theta_{2}^{\perp}+\left(-6 \alpha_{1}\left(1-\alpha_{1}-\alpha_{3}\right)+\left(1-\alpha_{3}\right) \alpha_{3}\right) \theta_{1}^{\perp}+\left(1-\alpha_{3}\right) \theta_{0}^{\perp}\right)
\end{gathered}
$$

where we have replaced $\alpha_{2}=1-\alpha_{1}-\alpha_{3}$ before the integration and we take $\xi=\bar{u}$ since the second quark is at the point $x=0$. The $q_{1}$ and $q_{2}$ indicate the quark components of the vector meson. The $\rho$ meson has both light quarks, hence $m_{q_{1}}=m_{q_{2}}=0$. The $K^{*}$ meson has one strange quark and one light quark, thus $m_{q_{1}}=m_{s}$ but $m_{q_{2}}=0$. The derived DA parameters are given as follows: 


$$
\begin{aligned}
& \psi_{0}^{\perp}=\zeta_{4}^{\perp} \\
& \tilde{\psi}_{0}^{\perp}=\tilde{\zeta}_{4}^{\perp} \\
& \theta_{0}^{\perp}=-(1 / 6) \kappa_{3}^{\perp}-(1 / 3) \kappa_{4}^{\perp} \\
& \tilde{\theta}_{0}^{\perp}=-(1 / 6) \kappa_{3}^{\perp}+(1 / 3) \kappa_{4}^{\perp} \\
& \phi_{0}^{\perp}=(1 / 6) \kappa_{3}^{\perp}+(1 / 3) \kappa_{4}^{\perp} \\
& \tilde{\phi}_{0}^{\perp}=(1 / 6) \kappa_{3}^{\perp}-(1 / 3) \kappa_{4}^{\perp} \\
& \phi_{1}^{\perp}=(9 / 44) a_{2}^{\perp}+(1 / 8) \omega_{3}^{\perp}+(63 / 220)\left\langle\left\langle Q^{(1)}\right\rangle\right\rangle \\
& -(119 / 44)\left\langle\left\langle Q^{(3)}\right\rangle\right\rangle \\
& \tilde{\phi}_{1}^{\perp}=-(9 / 44) a_{2}^{\perp}+(1 / 8) \omega_{3}^{\perp}-(63 / 220)\left\langle\left\langle Q^{(1)}\right\rangle\right\rangle \\
& -(35 / 44)\left\langle\left\langle Q^{(3)}\right\rangle\right\rangle \\
& \psi_{1}^{\perp}=(3 / 44) a_{2}^{\perp}+(1 / 12) \omega_{3}^{\perp}+(49 / 110)\left\langle\left\langle Q^{(1)}\right\rangle\right\rangle \\
& -(7 / 22)\left\langle\left\langle Q^{(3)}\right\rangle\right\rangle+(7 / 3)\left\langle\left\langle Q^{(5)}\right\rangle\right\rangle \\
& \tilde{\psi}_{1}^{\perp}=-(3 / 44) a_{2}^{\perp}+(1 / 12) \omega_{3}^{\perp}-(49 / 110)\left\langle\left\langle Q^{(1)}\right\rangle\right\rangle \\
& +(7 / 22)\left\langle\left\langle Q^{(3)}\right\rangle\right\rangle+(7 / 3)\left\langle\left\langle Q^{(5)}\right\rangle\right\rangle \\
& \psi \frac{\perp}{2}=-(3 / 22) a_{2}^{\perp}-(1 / 12) \omega_{3}^{\perp}+(28 / 55)\left\langle\left\langle Q^{(1)}\right\rangle\right\rangle \\
& +(7 / 11)\left\langle\left\langle Q^{(3)}\right\rangle\right\rangle+(14 / 3)\left\langle\left\langle Q^{(5)}\right\rangle\right\rangle \\
& \tilde{\psi}_{2}^{\perp}=(3 / 22) a_{2}^{\perp}-(1 / 12) \omega_{3}^{\perp}-(28 / 55)\left\langle\left\langle Q^{(1)}\right\rangle\right\rangle \\
& -(7 / 11)\left\langle\left\langle Q^{(3)}\right\rangle\right\rangle+(14 / 3)\left\langle\left\langle Q^{(5)}\right\rangle\right\rangle \\
& \theta_{1}^{\|}=-(7 / 10) a_{1}^{\|} \zeta_{4}^{\|} \\
& \theta_{2}^{\|}=(7 / 5) a_{1}^{\|} \zeta_{4}^{\|} \\
& \psi_{2}^{\|}=-(7 / 20) a_{1}^{\|} \zeta_{4}^{\|} \\
& \theta_{1}^{\perp}=-(21 / 10) \zeta_{4}^{\perp} a_{1}^{\perp} \\
& \tilde{\theta}_{1}^{\perp}=(21 / 10) \zeta_{4}^{\perp} a_{1}^{\perp} \\
& \theta_{2}^{\perp}=(21 / 5) \zeta_{4}^{\perp} a_{1}^{\perp}
\end{aligned}
$$

TABLE V. The numerical values for the parameters in the DAs for vector mesons $\rho$ and $K^{*}$. The renormalization scale is $\mu=1 \mathrm{GeV}$. The accuracy of these parameters are around $30 \%$ to $50 \%$.

\begin{tabular}{lccccccccc}
\hline \hline & $\rho^{ \pm}$ & $K^{*}$ & & $\rho^{ \pm}$ & $K^{*}$ & & $\rho^{ \pm}$ & $K^{*}$ \\
\hline$f_{V}[\mathrm{GeV}]$ & 0.216 & 0.22 & $\zeta_{3}^{\|}$ & 0.03 & 0.023 & $\omega_{3}^{\perp}$ & 0.55 & 0.3 \\
$f_{V}^{T}[\mathrm{GeV}]$ & 0.165 & 0.185 & $\tilde{\lambda}_{3}^{\|}$ & 0 & 0.035 & $\lambda_{3}^{\perp}$ & 0 & -0.025 \\
$m_{V}[\mathrm{GeV}]$ & 0.77 & 0.892 & $\tilde{\omega}_{3}^{\|}$ & -0.09 & -0.07 & $\zeta_{4}^{\|}$ & 0.07 & 0.02 \\
$a_{1}^{\|}$ & 0 & 0.03 & $\kappa_{3}^{\|}$ & 0 & 0 & $\tilde{\omega}_{4}^{\|}$ & -0.03 & -0.02 \\
$a_{1}^{\perp}$ & 0 & 0.04 & $\omega_{3}^{\|}$ & 0.15 & 0.1 & $\zeta_{4}^{\perp}$ & -0.08 & -0.05 \\
$a_{2}^{\|}$ & 0.15 & 0.11 & $\lambda_{3}^{\|}$ & 0 & -0.008 & $\tilde{\zeta}_{4}^{\perp}$ & -0.08 & -0.05 \\
$a_{2}^{\perp}$ & 0.14 & 0.1 & $\kappa_{3}^{\perp}$ & 0 & 0.003 & $\kappa_{4}^{\|}$ & 0 & -0.025 \\
& & & & & & $\kappa_{4}^{\perp}$ & 0 & 0.013 \\
\hline \hline
\end{tabular}

$$
\begin{gathered}
\tilde{\theta}_{2}^{\perp}=-(21 / 5) \zeta_{4}^{\perp} a_{1}^{\perp} \\
\tilde{\phi}_{2}^{\perp}=-(21 / 20) \zeta_{4}^{\perp} a_{1}^{\perp} \\
\left\langle\left\langle Q^{(1)}\right\rangle\right\rangle=-(10 / 3) \zeta_{4}^{\perp} \\
\left\langle\left\langle Q^{(3)}\right\rangle\right\rangle=-\zeta_{4}^{\perp} \\
\left\langle\left\langle Q^{(5)}\right\rangle\right\rangle=0
\end{gathered}
$$

The numerical values for the DA parameters are given in Table V.

The light quark masses are taken to be zero, namely, $m_{u}=m_{d}=0$, and the mass of the strange quark at $\mu=1 \mathrm{GeV}$ is taken to be $m_{s}=0.137 \mathrm{GeV}$.

\section{APPENDIX B: DETAILS OF CALCULATIONS IN THE THEORETICAL PART}

In this section, we present some steps of the calculations needed in the theoretical analysis. In what follows, $f(u)$ and $\mathcal{F}\left(\alpha_{i}\right)$ denote generic two- and three-particle DAs, respectively, and we let $K_{i}:=K_{i}\left(m_{Q} \sqrt{-x^{2}}\right) /\left(\sqrt{-x^{2}}\right)^{i}$ and $K_{j}:=K_{j}\left(m_{Q^{\prime}} \sqrt{-x^{2}}\right) /\left(\sqrt{-x^{2}}\right)^{j}$.

\section{Terms proportional to $\mathcal{O}\left(\langle G\rangle^{\mathbf{0}}\right)$}

For the sake of simplicity, we suppress the integral measures, $\int d u \int d^{4} x e^{i(p+\bar{u} q) x}$, on the left-hand side.

$$
K_{i} K_{j} f(u) \rightarrow \frac{i}{4} \frac{16 \pi^{2}}{\left(2 m_{Q}\right)^{i}\left(2 m_{Q^{\prime}}\right)^{j}}\left(M^{2}\right)^{i+j} f\left(u_{0}\right) e^{-m_{V}^{2} / 2 M^{2}} \int_{\left(m_{Q}+m_{Q^{\prime}}\right)^{2}}^{s_{0}} d s e^{-s / M^{2}} \times \int d \alpha \alpha^{i-1}(1-\alpha)^{j-1} \delta\left(s-\frac{m_{Q}^{2}}{\alpha}-\frac{m_{Q^{\prime}}^{2}}{1-\alpha}\right)
$$




$$
\begin{aligned}
x_{\mu} K_{i} K_{j} f(u) \rightarrow & \frac{-i\left(u_{0}^{-1} p_{\mu}+q_{\mu}\right)}{M^{2}} \frac{i}{4} \frac{16 \pi^{2}}{\left(2 m_{Q}\right)^{i}\left(2 m_{Q^{\prime}}\right)^{j}}\left(M^{2}\right)^{i+j} f\left(u_{0}\right) e^{-m_{V}^{2} / 2 M^{2}} \int_{\left(m_{\left.Q+m_{Q^{\prime}}\right)^{2}} s_{0}\right.} d s e^{-s / M^{2}} \\
& \times \int d \alpha \alpha^{i-1}(1-\alpha)^{j-1} \delta\left(s-\frac{m_{Q}^{2}}{\alpha}-\frac{m_{Q^{\prime}}^{2}}{1-\alpha}\right) \\
x_{\mu} x_{\nu} K_{i} K_{j} f(u) \rightarrow & \frac{-\left(u_{0}^{-1} p_{\mu}+q_{\mu}\right)\left(u_{0}^{-1} p_{\nu}+q_{\nu}\right)-u_{0}^{-1} M^{2} g_{\mu \nu}}{M^{4}} \frac{i}{4} \frac{16 \pi^{2}}{\left(2 m_{Q}\right)^{i}\left(2 m_{Q^{\prime}}\right)^{j}}\left(M^{2}\right)^{i+j} f\left(u_{0}\right) e^{-m_{V}^{2} / 2 M^{2}} \\
& \times \int_{\left(m_{\left.Q+m_{Q^{\prime}}\right)^{2}} s_{0}\right.} d s e^{-s / M^{2}} \int d \alpha \alpha^{i-1}(1-\alpha)^{j-1} \delta\left(s-\frac{m_{Q}^{2}}{\alpha}-\frac{m_{Q^{\prime}}^{2}}{1-\alpha}\right)
\end{aligned}
$$

$$
\begin{aligned}
& x^{2} K_{i} K_{j} f(u) \rightarrow \frac{i}{4} \frac{16 \pi^{2}}{\left(2 m_{Q}\right)^{i}\left(2 m_{Q^{\prime}}\right)^{j}} M^{2} e^{-m_{V}^{2} / 2 M^{2}} f\left(u_{0}\right) \int_{\left(m_{\left.Q+m_{Q^{\prime}}\right)^{2}}\right.}^{s_{0}} d s e^{-s / M^{2}} \\
& \times \int d \alpha \alpha^{i-1}(1-\alpha)^{j-1} \delta\left(s-\frac{m_{Q}^{2}}{\alpha}-\frac{m_{Q^{\prime}}^{2}}{1-\alpha}\right)\left(M^{2}\right)^{-3+i+j} \times \frac{-4(-1+\alpha)\left(M^{2}(-1+i+j) \alpha+m_{Q}^{2}\right)+4 \alpha m_{Q^{\prime}}^{2}}{\alpha(\alpha-1)} \\
& x_{\mu} x^{2} K_{i} K_{j} f(u) \rightarrow \frac{-i\left(u_{0}^{-1} p_{\mu}+q_{\mu}\right)}{M^{2}} \frac{i}{4} \frac{16 \pi^{2}}{\left(2 m_{Q}\right)^{i}\left(2 m_{Q^{\prime}}\right)^{j}} M^{2} e^{-m_{V}^{2} / 2 M^{2}} f\left(u_{0}\right) \int_{\left(m_{Q}+m_{Q^{\prime}}\right)^{2}}^{s_{0}} d s e^{-s / M^{2}} \\
& \times \int d \alpha \alpha^{i-1}(1-\alpha)^{j-1} \delta\left(s-\frac{m_{Q}^{2}}{\alpha}-\frac{m_{Q^{\prime}}^{2}}{1-\alpha}\right)\left(M^{2}\right)^{-3+i+j} \\
& \times \frac{-4(-1+\alpha)\left(M^{2}(-1+i+j) \alpha+m_{Q}^{2}\right)+4 \alpha m_{Q^{\prime}}^{2}}{\alpha(\alpha-1)} \\
& x_{\mu} x_{\nu} x^{2} K_{i} K_{j} f(u) \rightarrow \frac{-\left(u_{0}^{-1} p_{\mu}+q_{\mu}\right)\left(u_{0}^{-1} p_{\nu}+q_{\nu}\right)-u_{0}^{-1} M^{2} g_{\mu \nu}}{M^{4}} \frac{i}{4} \frac{16 \pi^{2}}{\left(2 m_{Q}\right)^{i}\left(2 m_{Q^{\prime}}\right)^{j}} M^{2} e^{-m_{V}^{2} / 2 M^{2}} f\left(u_{0}\right) \\
& \times \int_{\left(m_{Q}+m_{Q^{\prime}}\right)^{2}}^{s_{0}} d s e^{-s / M^{2}} \int d \alpha \alpha^{i-1}(1-\alpha)^{j-1} \delta\left(s-\frac{m_{Q}^{2}}{\alpha}-\frac{m_{Q^{\prime}}^{2}}{1-\alpha}\right) \\
& \times\left(M^{2}\right)^{-3+i+j} \frac{-4(-1+\alpha)\left(M^{2}(-1+i+j) \alpha+m_{Q}^{2}\right)+4 \alpha m_{Q^{\prime}}^{2}}{\alpha(\alpha-1)}
\end{aligned}
$$

$$
\begin{aligned}
x^{4} K_{i} K_{j} f(u) \rightarrow & \frac{i}{4} \frac{16 \pi^{2}}{\left(2 m_{Q}\right)^{i}\left(2 m_{Q^{\prime}}\right)^{j}} M^{2} e^{-m_{V}^{2} / 2 M^{2}} f\left(u_{0}\right) \int_{\left(m_{\left.Q^{+}+m_{Q^{\prime}}\right)^{2}}\right.}^{s_{0}} d s e^{-s / M^{2}} \\
& \times \int d \alpha \alpha^{i-1}(1-\alpha)^{j-1} \delta\left(s-\frac{m_{Q}^{2}}{\alpha}-\frac{m_{Q^{\prime}}^{2}}{1-\alpha} \frac{16\left(M^{2}\right)^{-5+i+j}}{\alpha^{2}(-1+\alpha)^{2}} \times\left(M^{4}(-2+i+j)(-1+i+j)(-1+\alpha)^{2} \alpha^{2}\right.\right. \\
& \left.+(-1+\alpha)^{2} m_{Q}^{2}\left(2 M^{2}(-2+i+j) \alpha+m_{Q}^{2}\right)-2(-1+\alpha) \alpha\left(M^{2}(-2+i+j) \alpha+m_{Q}^{2}\right) m_{Q^{\prime}}^{2}+\alpha^{2} m_{Q^{\prime}}^{4}\right)
\end{aligned}
$$

$$
\begin{aligned}
x_{\mu} x^{4} K_{i} K_{j} f(u) \rightarrow & \frac{-i\left(u_{0}^{-1} p_{\mu}+q_{\mu}\right)}{M^{2}} \frac{i}{4} \frac{16 \pi^{2}}{\left(2 m_{Q}\right)^{i}\left(2 m_{Q^{\prime}}\right)^{j}} M^{2} e^{-m_{V}^{2} / 2 M^{2}} f\left(u_{0}\right) \int_{\left(m_{\left.Q^{+}+m_{Q^{\prime}}\right)^{2}} s_{0}\right.} d s e^{-s / M^{2}} \\
& \times \int d \alpha \alpha^{i-1}(1-\alpha)^{j-1} \delta\left(s-\frac{m_{Q}^{2}}{\alpha}-\frac{m_{Q^{\prime}}^{2}}{1-\alpha}\right) \frac{16\left(M^{2}\right)^{-5+i+j}}{\alpha^{2}(-1+\alpha)^{2}} \times\left(M^{4}(-2+i+j)(-1+i+j)(-1+\alpha)^{2} \alpha^{2}\right. \\
& \left.+(-1+\alpha)^{2} m_{Q}^{2}\left(2 M^{2}(-2+i+j) \alpha+m_{Q}^{2}\right)-2(-1+\alpha) \alpha\left(M^{2}(-2+i+j) \alpha+m_{Q}^{2}\right) m_{Q^{\prime}}^{2}+\alpha^{2} m_{Q^{\prime}}^{4}\right)
\end{aligned}
$$




$$
\begin{aligned}
x_{\mu} x_{\nu} x^{4} K_{i} K_{j} f(u) \rightarrow & \frac{-\left(u_{0}^{-1} p_{\mu}+q_{\mu}\right)\left(u_{0}^{-1} p_{\nu}+q_{\nu}\right)-u_{0}^{-1} M^{2} g_{\mu \nu}}{M^{4}} \frac{i}{4} \frac{16 \pi^{2}}{\left(2 m_{Q}\right)^{i}\left(2 m_{Q^{\prime}}\right)^{j}} M^{2} e^{-m_{V}^{2} / 2 M^{2}} f\left(u_{0}\right) \\
& \times \int_{\left(m_{\left.Q^{+}+m_{Q^{\prime}}\right)^{2}} s_{0}\right.} d s e^{-s / M^{2}} \int d \alpha \alpha^{i-1}(1-\alpha)^{j-1} \delta\left(s-\frac{m_{Q}^{2}}{\alpha}-\frac{m_{Q^{\prime}}^{2}}{1-\alpha}\right) \\
& \times \frac{16\left(M^{2}\right)^{-5+i+j}}{\alpha^{2}(-1+\alpha)^{2}}\left(M^{4}(-2+i+j)(-1+i+j)(-1+\alpha)^{2} \alpha^{2}\right. \\
& +(-1+\alpha)^{2} m_{Q}^{2}\left(2 M^{2}(-2+i+j) \alpha+m_{Q}^{2}\right) \\
& \left.-2(-1+\alpha) \alpha\left(M^{2}(-2+i+j) \alpha+m_{Q}^{2}\right) m_{Q^{\prime}}^{2}+\alpha^{2} m_{Q^{\prime}}^{4}\right) .
\end{aligned}
$$

\section{Terms proportional to $\mathcal{O}\left(\langle G\rangle^{\mathbf{1}}\right)$}

For the sake of simplicity, we suppress the integral measures, $\int d u \int d^{4} x \int \mathcal{D} \alpha_{i} e^{i\left(p+\left(\alpha_{1}+u \alpha_{3}\right) q\right) x}$, on the left-hand side.

$$
\begin{aligned}
& K_{i} K_{j} f(u) \mathcal{F}\left(\alpha_{i}\right) \rightarrow \frac{i}{4} \frac{16 \pi^{2}}{\left(2 m_{Q}\right)^{i}\left(2 m_{Q^{\prime}}\right)^{j}}\left(M^{2}\right)^{i+j} e^{-m_{V}^{2} / 2 M^{2}} \int_{\left(m_{Q}+m_{Q^{\prime}}\right)^{2}}^{s_{0}} d s e^{-s / M^{2}} \\
& \times \int d \alpha \alpha^{i-1}(1-\alpha)^{j-1} \delta\left(s-\frac{m_{Q}^{2}}{\alpha}-\frac{m_{Q^{\prime}}^{2}}{1-\alpha}\right) \times \int_{0}^{1 / 2} d \alpha_{1} \int_{1 / 2-\alpha_{1}}^{1-\alpha_{1}} d \alpha_{3} f\left(\frac{u_{0}-\alpha_{1}}{\alpha_{3}}\right) \frac{F\left(\alpha_{i}\right)}{\alpha_{3}} \\
& x_{\mu} K_{i} K_{j} f(u) \mathcal{F}\left(\alpha_{i}\right) \rightarrow \frac{-i\left(u_{0}^{-1} p_{\mu}+q_{\mu}\right)}{M^{2}} \frac{i}{4} \frac{16 \pi^{2}}{\left(2 m_{Q}\right)^{i}\left(2 m_{Q^{\prime}}\right)^{j}}\left(M^{2}\right)^{i+j} e^{-m_{V}^{2} / 2 M^{2}} \\
& \times \int_{\left(m_{\left.Q+m_{Q^{\prime}}\right)^{2}}\right.}^{s_{0}} d s e^{-s / M^{2}} \int d \alpha \alpha^{i-1}(1-\alpha)^{j-1} \delta\left(s-\frac{m_{Q}^{2}}{\alpha}-\frac{m_{Q^{\prime}}^{2}}{1-\alpha}\right) \\
& \times \int_{0}^{1 / 2} d \alpha_{1} \int_{1 / 2-\alpha_{1}}^{1-\alpha_{1}} d \alpha_{3} f\left(\frac{u_{0}-\alpha_{1}}{\alpha_{3}}\right) \frac{\mathcal{F}\left(\alpha_{i}\right)}{\alpha_{3}} \\
& x_{\mu} x_{\nu} K_{i} K_{j} f(u) \mathcal{F}\left(\alpha_{i}\right) \rightarrow \frac{-\left(u_{0}^{-1} p_{\mu}+q_{\mu}\right)\left(u_{0}^{-1} p_{\nu}+q_{\nu}\right)-u_{0}^{-1} M^{2} g_{\mu \nu}}{M^{4}} \frac{i}{4} \frac{16 \pi^{2}}{\left(2 m_{Q}\right)^{i}\left(2 m_{Q^{\prime}}\right)^{j}}\left(M^{2}\right)^{i+j} e^{-m_{V}^{2} / 2 M^{2}} \\
& \times \int_{\left(m_{Q}+m_{Q^{\prime}}\right)^{2}}^{s_{0}} d s e^{-s / M^{2}} \int d \alpha \alpha^{i-1}(1-\alpha)^{j-1} \delta\left(s-\frac{m_{Q}^{2}}{\alpha}-\frac{m_{Q^{\prime}}^{2}}{1-\alpha}\right) \\
& \times \int_{0}^{1 / 2} d \alpha_{1} \int_{1 / 2-\alpha_{1}}^{1-\alpha_{1}} d \alpha_{3} f\left(\frac{u_{0}-\alpha_{1}}{\alpha_{3}}\right) \frac{\mathcal{F}\left(\alpha_{i}\right)}{\alpha_{3}} \\
& x^{2} K_{i} K_{j} f(u) \mathcal{F}\left(\alpha_{i}\right) \rightarrow \frac{i}{4} \frac{16 \pi^{2}}{\left(2 m_{Q}\right)^{i}\left(2 m_{Q^{\prime}}\right)^{j}} e^{-m_{V}^{2} / 2 M^{2}} M^{2} \int_{\left(m_{Q}+m_{Q^{\prime}}\right)^{2}}^{s_{0}} d s e^{-s / M^{2}} \\
& \times \int d \alpha \alpha^{i-1}(1-\alpha)^{j-1} \delta\left(s-\frac{m_{Q}^{2}}{\alpha}-\frac{m_{Q^{\prime}}^{2}}{1-\alpha}\right)\left(M^{2}\right)^{-3+i+j} \\
& \times \frac{-4(-1+\alpha)\left(M^{2}(-1+i+j) \alpha+m_{Q}^{2}\right)+4 \alpha m_{Q^{\prime}}^{2}}{(-1+\alpha) \alpha} \\
& \times \int_{0}^{1 / 2} d \alpha_{1} \int_{1 / 2-\alpha_{1}}^{1-\alpha_{1}} d \alpha_{3} f\left(\frac{u_{0}-\alpha_{1}}{\alpha_{3}}\right) \frac{\mathcal{F}\left(\alpha_{i}\right)}{\alpha_{3}}
\end{aligned}
$$




$$
\begin{aligned}
x_{\mu} x^{2} K_{i} K_{j} f(u) F\left(\alpha_{i}\right) \rightarrow & \frac{-i\left(u_{0} p_{\mu}+q_{\mu}\right)}{M^{2}} \frac{i}{4} \frac{16 \pi^{2}}{\left(2 m_{Q}\right)^{i}\left(2 m_{Q^{\prime}}\right)^{j}} e^{-m_{V}^{2} / 2 M^{2}} M^{2} \int_{\left(m_{Q}+m_{Q^{\prime}}\right)^{2}}^{s_{0}} d s e^{-s / M^{2}} \int d \alpha \alpha^{i-1}(1-\alpha)^{j-1} \\
& \times \delta\left(s-\frac{m_{Q}^{2}}{\alpha}-\frac{m_{Q^{\prime}}^{2}}{1-\alpha}\right)\left(M^{2}\right)^{-3+i+j} \frac{-4(-1+\alpha)\left(M^{2}(-1+i+j) \alpha+m_{Q}^{2}\right)+4 \alpha m_{Q^{\prime}}^{2}}{(-1+\alpha) \alpha} \\
& \times \int_{0}^{1 / 2} d \alpha_{1} \int_{1 / 2-\alpha_{1}}^{1-\alpha_{1}} d \alpha_{3} f\left(\frac{u_{0}-\alpha_{1}}{\alpha_{3}}\right) \frac{F\left(\alpha_{i}\right)}{\alpha_{3}} \\
x_{\mu} x_{\nu} x^{2} K_{i} K_{j} f(u) \mathcal{F}\left(\alpha_{i}\right) \rightarrow & \frac{-\left(u_{0}^{-1} p_{\mu}+q_{\mu}\right)\left(u_{0}^{-1} p_{\nu}+q_{\nu}\right)-u_{0}^{-1} M^{2} g_{\mu \nu}}{M^{4}} \frac{i}{4} \frac{16 \pi^{2}}{\left(2 m_{Q}\right)^{i}\left(2 m_{Q^{\prime}}\right)^{j}} e^{-m_{V}^{2} / 2 M^{2}} M^{2} \\
& \times \int_{\left(m_{Q}+m_{Q^{\prime}}\right)^{2}}^{s_{0}} d s e^{-s / M^{2}} \int d \alpha \alpha^{i-1}(1-\alpha)^{j-1} \delta\left(s-\frac{m_{Q}^{2}}{\alpha}-\frac{m_{Q^{\prime}}^{2}}{1-\alpha}\right) \\
& \times\left(M^{2}\right)^{-3+i+j} \frac{-4(-1+\alpha)\left(M^{2}(-1+i+j) \alpha+m_{Q}^{2}\right)+4 \alpha m_{Q^{\prime}}^{2}}{(-1+\alpha) \alpha} \\
& \times \int_{0}^{1 / 2} d \alpha_{1} \int_{1 / 2-\alpha_{1}}^{1-\alpha_{1}} d \alpha_{3} f\left(\frac{u_{0}-\alpha_{1}}{\alpha_{3}}\right) \frac{\mathcal{F}\left(\alpha_{i}\right)}{\alpha_{3}}
\end{aligned}
$$

$$
\begin{aligned}
x^{4} K_{i} K_{j} f(u) \mathcal{F}\left(\alpha_{i}\right) \rightarrow & \frac{i}{4} \frac{16 \pi^{2}}{\left(2 m_{Q}\right)^{i}\left(2 m_{Q^{\prime}}\right)^{j}} M^{2} e^{-m_{V}^{2} / 2 M^{2}} \int_{\left(m_{Q}+m_{Q^{\prime}}\right)^{2}}^{s_{0}} d s e^{-s / M^{2}} \\
& \times \int d \alpha \alpha^{i-1}(1-\alpha)^{j-1} \delta\left(s-\frac{m_{Q}^{2}}{\alpha}-\frac{m_{Q^{\prime}}^{2}}{1-\alpha}\right) \frac{16\left(M^{2}\right)^{-5+i+j}}{(-1+\alpha)^{2} \alpha^{2}} \\
& \times\left(M^{4}(-2+i+j)(-1+i+j)(-1+\alpha)^{2} \alpha^{2}+(-1+\alpha)^{2} m_{Q}^{2}\left(2 M^{2}(-2+i+j) \alpha+m_{Q}^{2}\right)\right. \\
& \left.-2(-1+\alpha) \alpha\left(M^{2}(-2+i+j) \alpha+m_{Q}^{2}\right) m_{Q^{\prime}}^{2}+\alpha m_{Q^{\prime}}^{4}\right) \int_{0}^{1 / 2} d \alpha_{1} \int_{1 / 2-\alpha_{1}}^{1-\alpha_{1}} d \alpha_{3} f\left(\frac{u_{0}-\alpha_{1}}{\alpha_{3}}\right) \frac{\mathcal{F}\left(\alpha_{i}\right)}{\alpha_{3}}
\end{aligned}
$$

$$
\begin{aligned}
x_{\mu} x^{4} K_{i} K_{j} f(u) \mathcal{F}\left(\alpha_{i}\right) \rightarrow & \frac{-i\left(u_{0}^{-1} p_{\mu}+q_{\mu}\right)}{M^{2}} \frac{i}{4} \frac{16 \pi^{2}}{\left(2 m_{Q}\right)^{i}\left(2 m_{Q^{\prime}}\right)^{j}} M^{2} e^{-m_{V}^{2} / 2 M^{2}} \int_{\left(m_{\left.Q^{+}+m_{Q^{\prime}}\right)^{2}} s_{0}\right.} d s e^{-s / M^{2}} \\
& \times \int d \alpha \alpha^{i-1}(1-\alpha)^{j-1} \delta\left(s-\frac{m_{Q}^{2}}{\alpha}-\frac{m_{Q^{\prime}}^{2}}{1-\alpha}\right) \frac{16\left(M^{2}\right)^{-5+i+j}}{(-1+\alpha)^{2} \alpha^{2}} \\
& \times\left(M^{4}(-2+i+j)(-1+i+j)(-1+\alpha)^{2} \alpha^{2}+(-1+\alpha)^{2} m_{Q}^{2}\left(2 M^{2}(-2+i+j) \alpha+m_{Q}^{2}\right)\right. \\
& \left.-2(-1+\alpha) \alpha\left(M^{2}(-2+i+j) \alpha+m_{Q}^{2}\right) m_{Q^{\prime}}^{2}+\alpha m_{Q^{\prime}}^{4}\right) \times \int_{0}^{1 / 2} d \alpha_{1} \int_{1 / 2-\alpha_{1}}^{1-\alpha_{1}} d \alpha_{3} f\left(\frac{u_{0}-\alpha_{1}}{\alpha_{3}}\right) \frac{\mathcal{F}\left(\alpha_{i}\right)}{\alpha_{3}}
\end{aligned}
$$

$$
\begin{aligned}
x_{\mu} x_{\nu} x^{4} K_{i} K_{j} f(u) \mathcal{F}\left(\alpha_{i}\right) \rightarrow & \frac{-\left(u_{0}^{-1} p_{\mu}+q_{\mu}\right)\left(u_{0}^{-1} p_{\nu}+q_{\nu}\right)-u_{0}^{-1} M^{2} g_{\mu \nu}}{M^{4}} \frac{i}{4} \frac{16 \pi^{2}}{\left(2 m_{Q}\right)^{i}\left(2 m_{Q^{\prime}}\right)^{j}} M^{2} e^{-m_{V}^{2} / 2 M^{2}} \\
& \times \int_{\left(m_{Q}+m_{Q^{\prime}}\right)^{2}}^{s_{0}} d s e^{-s / M^{2}} \int d \alpha \alpha^{i-1}(1-\alpha)^{j-1} \delta\left(s-\frac{m_{Q}^{2}}{\alpha}-\frac{m_{Q^{\prime}}^{2}}{1-\alpha}\right) \\
& \times \frac{16\left(M^{2}\right)^{-5+i+j}}{(-1+\alpha)^{2} \alpha^{2}}\left(M^{4}(-2+i+j)(-1+i+j)(-1+\alpha)^{2} \alpha^{2}\right. \\
& \left.+(-1+\alpha)^{2} m_{Q}^{2}\left(2 M^{2}(-2+i+j) \alpha+m_{Q}^{2}\right)-2(-1+\alpha) \alpha\left(M^{2}(-2+i+j) \alpha+m_{Q}^{2}\right) m_{Q^{\prime}}^{2}+\alpha m_{Q^{\prime}}^{4}\right) \\
& \times \int_{0}^{1 / 2} d \alpha_{1} \int_{1 / 2-\alpha_{1}}^{1-\alpha_{1}} d \alpha_{3} f\left(\frac{u_{0}-\alpha_{1}}{\alpha_{3}}\right) \frac{\mathcal{F}\left(\alpha_{i}\right)}{\alpha_{3}}
\end{aligned}
$$


where

$$
u_{0}:=\frac{M_{1}^{2}}{M_{1}^{2}+M_{2}^{2}}
$$

For terms containing $q \cdot x$, we perform the following operation:

$$
\begin{gathered}
(q \cdot x)^{n} f(u) \rightarrow \begin{cases}\left(-i \frac{\partial}{\partial u}\right)^{n} f(u), & n>0 \\
i^{-n} \int_{0}^{u} d v_{n} \cdots \int_{0}^{v_{3}} d v_{2} \int_{0}^{v_{2}} d v_{1} f\left(v_{1}\right), & n<0\end{cases} \\
(q \cdot x)^{n} \mathcal{F}\left(\alpha_{i}\right) \rightarrow \begin{cases}\left(\frac{i}{u} \frac{\partial}{\partial \alpha_{3}}\right)^{n} \mathcal{F}\left(\alpha_{i}\right), & n>0 \\
(-i u)^{-n} \int_{0}^{\alpha_{3}} d \alpha_{3}^{(n)} \cdots \int_{0}^{\alpha_{3}^{(3)}} d \alpha_{3}^{(2)} \int_{0}^{\alpha_{3}^{(2)}} d \alpha_{3}^{(1)} \mathcal{F}\left(\alpha_{1}, 1-\alpha_{1}-\alpha_{3}^{(1)}, \alpha_{3}^{(1)}\right), & n<0\end{cases}
\end{gathered}
$$

[1] M. Mattson et al. (SELEX Collaboration), Phys. Rev. Lett. 89, 112001 (2002).

[2] A. Ocherashvili et al. (SELEX Collaboration), Phys. Lett. B 628, 18 (2005).

[3] R. Aaij et al. (LHCb Collaboration), Phys. Rev. Lett. 119, 112001 (2017).

[4] R. Aaij et al. (LHCb Collaboration), Phys. Rev. Lett. 121, 162002 (2018).

[5] M. A. Shifman, A. I. Vainshtein, and V. I. Zakharov, Nucl. Phys. B147, 385 (1979).

[6] I. I. Balitsky, V. M. Braun, and A. V. Kolesnichenko, Nucl. Phys. B312, 509 (1989).

[7] V. M. Braun and I. E. Filyanov, Z. Phys. C 44, 157 (1989).

[8] V. L. Chernyak and I. R. Zhitnitsky, Nucl. Phys. B345, 137 (1990).

[9] V. M. Braun, in Rostock 97, Progress in Heavy Quark Physics, edited by M. Beyer, T. Mannel, and H. Schröder (Rostock University, Rostock, 1997), p. 105.

[10] V. M. Braun, Proc. Sci., hf8 (1999) 006 [arXiv:hep-ph/ 9911206].

[11] P. Colangelo and A. Khodjamirian, in At the Frontier of Particle Physics, Vol. 3, edited by M. Shifman (World Scientific, Singapore, 2001), p. 1495.

[12] V. M. Belyaev, V. M. BraunA. Khodjamirian, and R. Rückl, Phys. Rev. D 51, 6177 (1995).

[13] Z. S. Brown, W. Detmold, S. Meinel, and K. Orginos, Phys. Rev. D 90, 094507 (2014).

[14] E. Hernandez, J. Nieves, and J. M. Verde-Velasco, Phys. Lett. B 663, 234 (2008).
[15] J. M. Flynn, E. Hernandez, and J. Nieves, Phys. Rev. D 85, 014012 (2012).

[16] T. M. Aliev, K. Azizi, and M. Savcı, Nucl. Phys. A895, 59 (2012).

[17] T. M. Aliev, K. Azizi, and M. Savc1, J. Phys. G 40, 065003 (2013).

[18] K. Azizi, T. M. Aliev, and M. Savcı, J. Phys. Conf. Ser. 556, 012016 (2014).

[19] K. Azizi, A. R. Olamaei, and S. Rostami, Eur. Phys. J. A 54, 162 (2018).

[20] A. R. Olamaei, K. Azizi, and S. Rostami, Eur. Phys. J. C 80, 613 (2020).

[21] S. Rostami, K. Azizi, and A. R. Olamaei, Chin. Phys. C 45, 023120 (2021).

[22] H. I. Alrebdi, T. M. Aliev, and K. Şimşek, Phys. Rev. D 102, 074007 (2020).

[23] T. M. Aliev and K. Şimşek, Eur. Phys. J. C 80, 976 (2020).

[24] K. Azizi, A. R. Olamaei, and S. Rostami, Eur. Phys. J. C 80, 1196 (2020).

[25] H. F. Jones and M. D. Scadron, Ann. Phys. (N.Y.) 81, 1 (1973).

[26] P. Ball, V. Braun, Y. Koike, and K. Tanaka, Nucl. Phys. B529, 323 (1998).

[27] P. Ball and V. Braun, Nucl. Phys. B543, 201 (1999).

[28] P. Ball and V. M. Braun, Phys. Rev. D 54, 2182 (1996).

[29] P. Ball, V. M. Braun, and A. Lenz, J. High Energy Phys. 05 (2006) 004.

[30] P. Ball, J. High Energy Phys. 01 (1999) 010.

[31] P. Ball and R. Zwicky, Phys. Rev. D 71, 014015 (2005).

[32] H. H. Patel, Comput. Phys. Commun. 197, 276 (2015). 